\title{
Qualitative Simulation of Genetic Regulatory Networks Using Piecewise-Linear Models
}

\author{
Hidde de Jong ${ }^{1}$, Jean-Luc Gouzé ${ }^{2}$, Céline HernandeZ ${ }^{3}$, \\ MiChEL PAGE $^{1,4}$, TEWFIK SARI ${ }^{5}$ AND JOHANNES GEISELMANN ${ }^{6}$ \\ ${ }^{1}$ Institut National de Recherche en Informatique et en Automatique (INRIA), Unité de \\ recherche Rhône-Alpes, 655 avenue de l'Europe, Montbonnot, 38334 Saint Ismier \\ Cedex, France. \\ ${ }^{2}$ Institut National de Recherche en Informatique et en Automatique (INRIA), Unité de \\ recherche Sophia Antipolis, 2004 route des Lucioles, BP 93, 06902 Sophia Antipolis, \\ France. \\ ${ }^{3}$ Swiss Institute of Bioinformatics (SIB), CMU - Rue Michel-Servet 1, 1211 Geneva 4, \\ Switzerland. \\ ${ }^{4}$ École Supérieure des Affaires, Université Pierre Mendès France, Grenoble, France. \\ ${ }^{5}$ Laboratoire de Mathématiques, 4 rue des Frères Lumière, Université de Haute \\ Alsace, 68093 Mulhouse, France. \\ ${ }^{6}$ Adaptation et Pathogénie des Microorganismes (CNRS FRE 2620), Université Joseph \\ Fourier, 460 rue de la Piscine, BP53, 38041 Grenoble Cedex 9, France.
}

\begin{abstract}
In order to cope with the large amounts of data that have become available in genomics, mathematical tools for the analysis of networks of interactions between genes, proteins, and other molecules are indispensable. We present a method for the qualitative simulation of genetic regulatory networks, based on a class of piecewise-linear (PL) differential equations that has been well-studied in mathematical biology. The simulation method is well-adapted to state-of-the-art measurement techniques in genomics, which often provide qualitative and coarsegrained descriptions of genetic regulatory networks. Given a qualitative model of a genetic regulatory network, consisting of a system of PL differential equations and inequality constraints on the parameter values, the method produces a graph of qualitative states and transitions between qualitative states, summarizing the qualitative dynamics of the system. The qualitative simulation method has been implemented in Java in the computer tool Genetic Network Analyzer.
\end{abstract}

\section{Introduction}

Recent progress in genomics has provided us with experimental tools that hold great promises for unraveling the networks of regulatory interactions between genes, pro- 
teins, and small molecules which underlie the functioning of living organisms. On the one hand, these techniques allow protein-DNA and protein-protein interactions to be identified, thus providing insight into the structure of genetic regulatory systems $(e . g$., $[51,57])$. On the other hand, they allow the evolution of the state of the system to be characterized, by large-scale measurement of the level of gene expression and protein activity across time (e.g., $[43,72])$.

In order to cope with the large amounts of data that have thus become available, formal methods for the representation and analysis of genetic regulatory networks are indispensable. Mathematical models allow networks of interactions to be described in a precise and unambiguous manner, while a large variety of analysis and simulation techniques exists to systematically derive behavior predictions from the models. The application of formal methods, especially when supported by computer tools, may lead to a comprehension of the structure and functioning of large and complex networks of interactions that cannot be obtained through intuitive approaches alone $[18,44]$.

The use of formal methods to study regulatory networks is currently subject to two major constraints [8]. First of all, the biochemical reaction mechanisms underlying the interactions are usually not or incompletely known. This prevents the formulation of detailed kinetic models, such as those developed for the genetic switch controlling phage $\lambda$ growth [45] or the feedback mechanisms regulating tryptophan synthesis in E. coli [59]. A second constraint arises from the general absence of quantitative information on kinetic parameters and molecular concentrations. As a consequence, traditional methods for numerical analysis are difficult to apply.

Few of the modeling and simulation methods that have been developed thus far are capable of handling the above constraints. A notable exception is formed by approaches based on a class of piecewise-linear $(P L)$ differential equation models originally proposed by Glass and Kauffman [27]. The state variables in the PL models correspond to the concentrations of proteins encoded by genes in the network, while the differential equations represent the interactions arising from the regulatory influence of some proteins on the synthesis and degradation of others. The regulatory interactions are modeled by means of step functions, which gives rise to the piecewise-linear structure of the differential equations. The use of step functions is motivated by the nonlinear, switch-like character of many of the interactions in gene expression and proteolysis $[56,71]$.

The PL models provide a coarse-grained description of genetic regulatory networks, well-adapted to state-of-the-art measurement techniques in genomics. Furthermore, the models have mathematical properties that favour qualitative analysis of the steady-state and transient behavior of regulatory systems [14, 15, 16, 24, 25, 28, 29, $31,41,47,48,52,60,61]$. On a formal level, the PL models are related to a class of asynchronous logical models proposed by Thomas and colleagues [66, 67]. PL models and their logical relatives have been used for the study of a number of prokaryotic and eukaryotic regulatory networks $[1,22,23,46,50,55,58,65]$. In addition, they have been used for modeling food webs [53], neural networks [42], and biological computers [6]. 
The use of step functions in PL models of genetic regulatory networks brings about some nontrivial mathematical problems. In particular, it involves the subdivision of the phase space into regions at the boundaries of which discontinuities may occur. Existing approaches either avoid these problems by restricting the analysis to a subclass of regulatory networks, or adopt solutions that have undesirable consequences for the predictiveness of the method. Recently, it has been shown that an approach capable of dealing with differential equations with discontinuous right-hand sides, widely used in control theory, allows the above-mentioned problems to be resolved in a mathematically proper and practically useful manner [31]. This approach, originally proposed by Filippov [19], is based on the generalization of the differential equations to differential inclusions.

In this paper we present a method for the qualitative simulation of genetic regulatory networks described by the generalized PL models. The method is obtained by formulating the analysis of PL models in terms of concepts developed for the qualitative simulation of dynamical systems $[12,39,40]$. The qualitative simulation method has been implemented in a publicly-available computer tool, called Genetic Network Analyzer (GNA) [10]. In the accompanying paper [9], we use the method and the tool to analyze a genetic regulatory network of biological interest, consisting of the genes and interactions that regulate the initiation of sporulation in Bacillus subtilis. The application shows that the simulation method can help to gain insight into the qualitative dynamics of complex regulatory networks involving a dozen of genes.

Two qualitative abstractions lie at the basis of this approach. First, we give a description of the dynamics of the system in terms of a graph of qualitative states and transitions between qualitative states. A qualitative state corresponds to a region in the phase space where the system behaves in a qualitatively distinct way. There exists a transition between two qualitative states, corresponding to contiguous regions in the phase space, if a solution starting in the first region reaches the second region, without passing through a third region. Second, instead of specifying numerical values for the parameters, we supplement the differential equations with inequality constraints that can usually be inferred from available biological data. The resulting qualitative $P L$ model corresponds to a region in the parameter space where, under certain conditions to be specified, the behavior of the system is described by the same graph of qualitative states and transitions between qualitative states. Given a qualitative PL model and an initial qualitative state, the simulation method determines all qualitative states that are reachable from the initial state through one or more transitions. The simulation is guaranteed to cover all possible solutions of the quantitative PL models subsumed by the qualitative PL model.

In the next section of the paper, the modeling of genetic regulatory networks by means of PL models will be discussed. The mathematical analysis of these models will be reviewed in Section 3. Sections 4 and 5 introduce a qualitative description of the dynamics of PL models and the notion of qualitative PL model, respectively. The qualitative simulation algorithm is detailed in Section 6 , followed by an investigation of its formal properties in Section 7. In the final section of the paper, the method is discussed in the context of related work. 


\section{Piecewise-linear models of genetic regulatory networks}

Figure 1 shows an example of a simple genetic regulatory network. The genes $a$ and $b$, transcribed from separate promoters, encode the proteins $\mathrm{A}$ and $\mathrm{B}$, each of which controls the expression of both genes. ${ }^{*}$ Proteins A and B repress gene $a$ and $b$ at different concentrations. Repression of the genes is achieved by binding of the proteins to regulatory sites overlapping with the promoters. The pattern of interactions gives rise to one positive and two negative feedback loops.

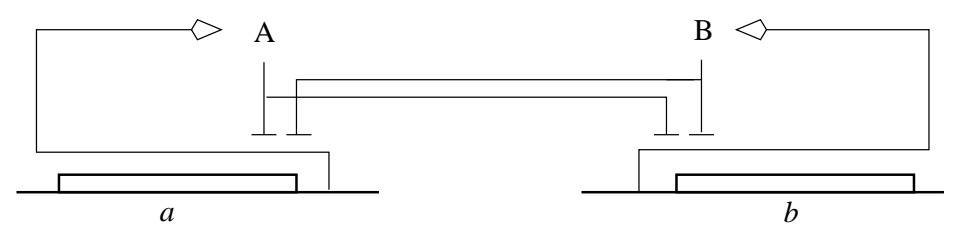

Figure 1: Example of a genetic regulatory network of two genes ( $a$ and $b$ ) coding for a regulatory protein (A and B). The notation follows, in a somewhat simplified form, the graphical conventions proposed by Kohn [38].

The dynamics of genetic regulatory networks can be modeled by a class of differential equations proposed by Mestl et al. [47], extending previous work by Glass and Kauffman [27] (see also the work of Snoussi and Thomas [60, 66] and Ratner and Tchuarev [63]). The equations have the general form

$$
\dot{x}_{i}=f_{i}(\boldsymbol{x})-g_{i}(\boldsymbol{x}) x_{i}, \quad x_{i} \geq 0,1 \leq i \leq n,
$$

where $\boldsymbol{x}=\left(x_{1}, \ldots, x_{n}\right)^{\prime}$ is a vector of cellular protein concentrations. The state equations (1) define the rate of change of each concentration $x_{i}$ as the difference of the rate of synthesis $f_{i}(\boldsymbol{x})$ and the rate of degradation $g_{i}(\boldsymbol{x}) x_{i}$ of the protein. In vector notation, the system of differential equations (1) is written as

$$
\dot{\boldsymbol{x}}=\boldsymbol{f}(\boldsymbol{x})-\boldsymbol{g}(\boldsymbol{x}) \boldsymbol{x}
$$

with $\boldsymbol{f}=\left(f_{1}, \ldots, f_{n}\right)^{\prime}$ and $\boldsymbol{g}=\operatorname{diag}\left(g_{1}, \ldots, g_{n}\right)$.

The function $f_{i}: \mathbb{R}_{\geq 0}^{n} \rightarrow \mathbb{R}_{\geq 0}$ expresses how the rate of synthesis of the protein encoded by gene $i$ depends on the concentrations $\boldsymbol{x}$ of proteins in the cell. It is defined as

$$
f_{i}(\boldsymbol{x})=\sum_{l \in L} \kappa_{i l} b_{i l}(\boldsymbol{x}),
$$

where $\kappa_{i l}$ is a rate parameter $\left(\kappa_{i l}>0\right), b_{i l}: \mathbb{R}_{\geq 0}^{n} \rightarrow\{0,1\}$ a regulation function, and $L$ a possibly empty set of indices of regulation functions. The function $g_{i}$ describes the regulation of protein degradation. It is defined analogously to $f_{i}$, except that we demand that $g_{i}(\boldsymbol{x})$ is strictly positive. In addition, in order to formally distinguish degradation rates from synthesis rates, we will denote the former by $\gamma$ instead of $\kappa$.

* As a notational convention, names of genes are printed in italic and names of proteins start with a capital. 
State equation for gene $a: \dot{x}_{a}=\kappa_{a} s^{-}\left(x_{a}, \theta_{a}^{2}\right) s^{-}\left(x_{b}, \theta_{b}^{1}\right)-\gamma_{a} x_{a}$

State equation for gene $b$ : $\dot{x}_{b}=\kappa_{b} s^{-}\left(x_{a}, \theta_{a}^{1}\right) s^{-}\left(x_{b}, \theta_{b}^{2}\right)-\gamma_{b} x_{b}$

Figure 2: State equations for the network of Figure 1. We will assume the following parameter values: $\theta_{a}^{1}=4, \theta_{a}^{2}=8, \theta_{b}^{1}=4, \theta_{b}^{2}=8, \kappa_{a}=20, \kappa_{b}=20, \gamma_{a}=2$, and $\gamma_{b}=2$.

Notice that with the above definitions of $f_{i}$ and $g_{i}$, the state equations (1) and (2) are piecewise-linear $(P L)$.

A regulation function $b_{i l}$ describes the logic of gene regulation $[54,60]$. More precisely, it describes the conditions under which the protein encoded by gene $i$ is synthesized (degraded) at a rate $\kappa_{i l}\left(\gamma_{i l} x_{i}\right)$. These conditions are formulated as expressions of step functions $s^{+}, s^{-}: \mathbb{R}^{2} \rightarrow\{0,1\}$ :

$$
s^{+}\left(x_{j}, \theta_{j}\right)=\left\{\begin{array}{ll}
1, & x_{j}>\theta_{j}, \\
0, & x_{j}<\theta_{j},
\end{array} \quad \text { and } \quad s^{-}\left(x_{j}, \theta_{j}\right)=1-s^{+}\left(x_{j}, \theta_{j}\right)\right.
$$

where $x_{j}$ is an element of the state vector $\boldsymbol{x}$ and $\theta_{j}$ a constant denoting a threshold concentration $\left(\theta_{j}>0\right)$. Notice that step functions $s^{+}\left(x_{j}, \theta_{j}\right)$ and $s^{-}\left(x_{j}, \theta_{j}\right)$ are not defined for $x_{j}=\theta_{j}$, so neither are the regulation functions in which they occur. We will use regulation functions that are the arithmetic equivalent of logical functions, as described in [54].

The simplest example of a regulation function is $b_{i l}(\boldsymbol{x})=s^{+}\left(x_{j}, \theta_{j}\right)$, which evaluates to 1 , if the concentration $x_{j}$ is above its threshold $\theta_{j}$. Another example is $b_{i l}(\boldsymbol{x})=1-s^{+}\left(x_{j}, \theta_{j}\right) s^{+}\left(x_{k}, \theta_{k}\right)$, which evaluates to 1 , if either $x_{j}$ is below its threshold $\theta_{j}$ or $x_{k}$ below its threshold $\theta_{k}$. The use of step functions has been motivated by the observation that the activity of a gene, as a function of the concentration of a regulatory protein, often follows a steep sigmoidal curve $[56,71]$. That is, the activity of the gene changes in a switch-like manner at a threshold concentration of the regulatory protein.

In Figure 2 the state equations for the example network are shown. Gene $a$ is expressed at a rate $\kappa_{a}$, if the concentration of protein $\mathrm{A}$ is below its threshold $\theta_{a}^{2}$ and the concentration of protein $\mathrm{B}$ below its threshold $\theta_{b}^{1}$, that is, if the regulation function $b_{a}\left(\left(x_{a}, x_{b}\right)^{\prime}\right)=s^{-}\left(x_{a}, \theta_{a}^{2}\right) s^{-}\left(x_{b}, \theta_{b}^{1}\right)$ evaluates to 1 . Analogously, gene $b$ is expressed at a rate $\kappa_{b}$, if the concentration of protein $\mathrm{A}$ is below the threshold $\theta_{a}^{1}$ and the concentration of protein B below the threshold $\theta_{b}^{2}\left(b_{b}\left(\left(x_{a}, x_{b}\right)^{\prime}\right)=s^{-}\left(x_{a}, \theta_{a}^{1}\right) s^{-}\left(x_{b}, \theta_{b}^{2}\right)=1\right)$. Degradation of the proteins $\mathrm{A}$ and $\mathrm{B}$ is assumed to be spontaneous, which gives rise to regulation functions having the value 1 , independent of the concentrations of the proteins.

The PL models can be extended to take into account input variables $\boldsymbol{u}=\left(u_{1}, \ldots\right.$, $\left.u_{m}\right)^{\prime}$, representing the concentration of proteins and small molecules whose synthesis and degradation are regulated outside the system. This leads to models of the form: 


$$
\dot{\boldsymbol{x}}=\boldsymbol{f}(\boldsymbol{x}, \boldsymbol{u})-\boldsymbol{g}(\boldsymbol{x}, \boldsymbol{u}) \boldsymbol{x} .
$$

In what follows, we will assume that the input variables are constant, i.e., $\dot{\boldsymbol{u}}=\mathbf{0}$. As a consequence, (5) can be reduced to (2) without loss of generality, by prior evaluation of the step function expressions in which input variables occur.

\section{Mathematical analysis of piecewise-linear models}

\subsection{Domains in phase space}

The dynamical properties of PL models of the form (2) can be analyzed in the $n$ dimensional phase space box $\Omega=\Omega_{1} \times \ldots \times \Omega_{n}$, where every $\Omega_{i}, 1 \leq i \leq n$, is defined as

$$
\Omega_{i}=\left\{x_{i} \in \mathbb{R}_{\geq 0} \mid 0 \leq x_{i} \leq \max _{i}\right\} .
$$

$\max _{i}$ is a parameter denoting a maximum concentration for the protein. It can be shown, by generalizing the argument in [26], that if we choose

$$
\max _{i}>\max _{\boldsymbol{x} \geq \mathbf{0}} f_{i}(\boldsymbol{x}) / g_{i}(\boldsymbol{x}),
$$

all trajectories starting inside $\Omega$ will remain in it, while trajectories starting outside will enter the phase space box at some point.

In general, a protein encoded by a gene will be involved in different interactions at different threshold concentrations, which after ordering are denoted by $\theta_{i}^{1}, \ldots, \theta_{i}^{p_{i}}$. The $(n-1)$-dimensional hyperplanes $x_{i}=\theta_{i}^{k_{i}}, 1 \leq k_{i} \leq p_{i}$, partition $\Omega$ into hyperrectangular regions that are called domains. Within each such region, the concentration of a protein equals a threshold or is bounded by thresholds. More precisely, a domain $D \subseteq \Omega$ is defined by $D=D_{1} \times \ldots \times D_{n}$, with every $D_{i}, 1 \leq i \leq n$, given by one of the following equations:

$$
\begin{aligned}
D_{i}= & \left\{x_{i} \mid 0 \leq x_{i}<\theta_{i}^{1}\right\}, \\
D_{i}= & \left\{x_{i} \mid x_{i}=\theta_{i}^{1}\right\}, \\
D_{i}= & \left\{x_{i} \mid \theta_{i}^{1}<x_{i}<\theta_{i}^{2}\right\}, \\
D_{i}= & \left\{x_{i} \mid x_{i}=\theta_{i}^{2}\right\}, \\
& \cdots \\
D_{i}= & \left\{x_{i} \mid x_{i}=\theta_{i}^{p_{i}}\right\}, \\
D_{i}= & \left\{x_{i} \mid \theta_{i}^{p_{i}}<x_{i} \leq \max _{i}\right\} .
\end{aligned}
$$

$\mathcal{D}$ denotes the set of all domains in $\Omega$. As can be easily verified, $|\mathcal{D}|=\prod_{i=1}^{n}\left(2 p_{i}+1\right)$.

We will distinguish two kinds of domain. A domain $D \in \mathcal{D}$ is called a regulatory domain, if there are no $i, j, 1 \leq i \leq n, 1 \leq j \leq p_{i}$, such that $D_{i}=\left\{x_{i} \mid x_{i}=\theta_{i}^{j}\right\}$. That is, in a regulatory domain none of the variables assumes a threshold value. On 
the other hand, $D \in \mathcal{D}$ is called a switching domain, if for at least one $i, 1 \leq i \leq n$, it holds that there is some $j, 1 \leq j \leq p_{i}$, such that $D_{i}=\left\{x_{i} \mid x_{i}=\bar{\theta}_{i}^{j}\right\}$. The corresponding variables $x_{i}$ are called switching variables. ${ }^{\dagger}$ The order of a switching domain is a number between 1 and $n$, equal to the number of switching variables. The sets of regulatory and switching domains are denoted by $\mathcal{D}_{r}$ and $\mathcal{D}_{s}$, respectively. We have $\left|\mathcal{D}_{r}\right|=\prod_{i=1}^{n}\left(p_{i}+1\right)$ and $\left|\mathcal{D}_{s}\right|=\prod_{i=1}^{n}\left(2 p_{i}+1\right)-\prod_{i=1}^{n}\left(p_{i}+1\right)$.

In Figure 3(a) the two-dimensional phase space box $\Omega$ for the example network is shown. As proteins A and B have two thresholds each, the phase space box is partitioned into 9 regulatory and 16 switching domains. An example of a regulatory domain is $D^{1}=\left\{\left(x_{a}, x_{b}\right)^{\prime} \in \mathbb{R}^{2} \mid 0 \leq x_{a}<\theta_{a}^{1}, 0 \leq x_{b}<\theta_{b}^{1}\right\}$, while $D^{4}=$ $\left\{\left(x_{a}, x_{b}\right)^{\prime} \in \mathbb{R}^{2} \mid 0 \leq x_{a}<\theta_{a}^{1}, x_{b}=\theta_{b}^{2}\right\}$ is an example of a switching domain. Notice that $x_{b}$ is the (only) switching variable in $D^{4}$.
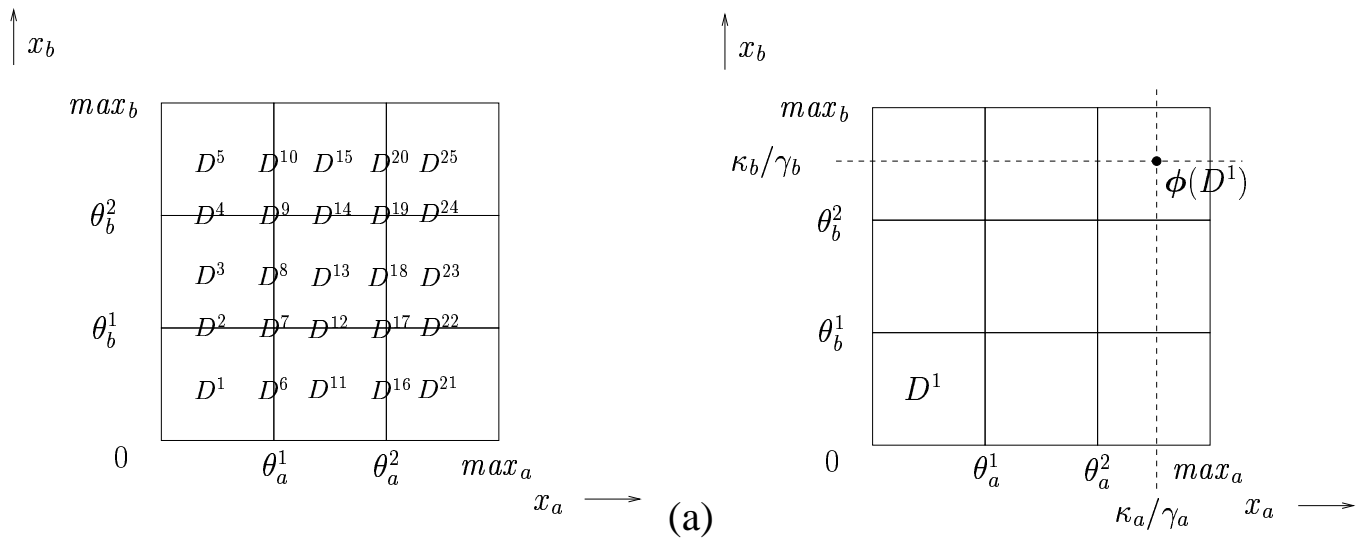

Figure 3: (a) Phase space box $\Omega$ for the PL model in Figure 2. (b) Target equilibrium $\phi\left(D^{1}\right)$ for the regulatory domain $D^{1}$. The variables $x_{a}$ and $x_{b}$ converge towards target equilibrium values $\kappa_{a} / \gamma_{a}$ and $\kappa_{b} / \gamma_{b}$, respectively. With the parameter values in Figure 2, the target equilibrium lies in the upperright domain.

As a preliminary step for the analysis of the dynamical properties of (2) in regulatory and switching domains, we define some auxiliary concepts. Let $D$ be a switching domain of order $k$, and $C$ the hyperplane of dimension $n-k$ containing $D$. The boundary of $D$ in $C$ is the set $B(D)$ of all points $\boldsymbol{x} \in C$, such that each ball $B_{C}(\boldsymbol{x}, \varepsilon)$ in $C$ of center $\boldsymbol{x}$ and radius $\varepsilon>0$ intersects both $D$ and $C \backslash D$ [37]. In the case that $D$ is a regulatory domain, $C$ equals $\Omega$. Now, for every $D \in \mathcal{D}$ we define the sets

$$
\begin{aligned}
& A(D)=\left\{D^{\prime} \in \mathcal{D}_{s} \mid D^{\prime} \subseteq B(D)\right\}, \text { and } \\
& R(D)=\left\{D^{\prime} \in \mathcal{D}_{r} \mid D \subseteq B\left(D^{\prime}\right)\right\}
\end{aligned}
$$

$A(D)$ contains the (switching) domains in the boundary of $D$, whereas $R(D)$ contains the regulatory domains that have $D$ in their boundary. In the case of the regulatory

${ }^{\dagger}$ In [47] switching domains are called $\Delta$-regions and switching variables are referred to as primary variables. 
domain $D^{1}$ in Figure 3, we find $A\left(D^{1}\right)=\left\{D^{2}, D^{6}, D^{7}\right\}$, while $A\left(D^{2}\right)=\left\{D^{7}\right\}$. Furthermore, $R\left(D^{1}\right)=\{\}$ and $R\left(D^{2}\right)=\left\{D^{1}, D^{3}\right\}$.

\subsection{Analysis in regulatory domains}

The behavior of systems described by a PL model of the form (2) has been wellcharacterized in the regulatory domains of $\Omega[25,52,60]$. When evaluating the step function expressions in (3) in a regulatory domain $D \in \mathcal{D}_{r}, f_{i}(\boldsymbol{x})$ reduces to some constant $\mu_{i}^{D} \in M_{i}$, and $g_{i}(\boldsymbol{x})$ to some constant $\nu_{i}^{D} \in N_{i}$, where

$$
\begin{gathered}
M_{i}=\left\{f_{i}(\boldsymbol{x}) \mid \mathbf{0} \leq \boldsymbol{x} \leq \boldsymbol{m a x}\right\}, \\
N_{i}=\left\{g_{i}(\boldsymbol{x}) \mid \boldsymbol{0} \leq \boldsymbol{x} \leq \boldsymbol{m a x}\right\} .
\end{gathered}
$$

More precisely, as a consequence of (3), $\mu_{i}^{D}$ and $\nu_{i}^{D}$ reduce to sums of rate constants $\kappa_{i l}$ and $\gamma_{i l}$, respectively. $M_{i}$ and $N_{i}$ collect the synthesis and degradation rates of the protein in different domains of $\Omega$. Inside a regulatory domain $D$, the state equations thus simplify to linear and uncoupled differential equations

$$
\dot{x}_{i}=\mu_{i}^{D}-\nu_{i}^{D} x_{i}, 1 \leq i \leq n,
$$

or, equivalently,

$$
\dot{\boldsymbol{x}}=\boldsymbol{\mu}^{D}-\boldsymbol{\nu}^{D} \boldsymbol{x},
$$

with $\boldsymbol{\mu}^{D}=\left(\mu_{1}^{D}, \ldots, \mu_{n}^{D}\right)^{\prime}$ and $\boldsymbol{\nu}^{D}=\operatorname{diag}\left(\nu_{1}^{D}, \ldots, \nu_{n}^{D}\right)$.

Let $\boldsymbol{\theta}=\left(\ldots, \theta_{i}^{k_{i}}, \ldots\right)^{\prime}, \boldsymbol{\kappa}=\left(\ldots, \kappa_{i l}, \ldots\right)^{\prime}$, and $\boldsymbol{\gamma}=\left(\gamma_{1}, \ldots, \gamma_{n}\right)^{\prime}$ be numerical parameter values. Furthermore, let $\boldsymbol{x}(0)=\boldsymbol{x}_{0}$ be a point in $\Omega$ representing the initial conditions. A continuously differentiable function $\boldsymbol{\xi}\left(t, \boldsymbol{x}_{0}, \boldsymbol{\theta}, \boldsymbol{\kappa}, \boldsymbol{\gamma}\right)$ is a solution of (11) on a time-interval $[0, \tau], \tau>0$, if $\boldsymbol{\xi}(0)=\boldsymbol{x}_{0}$ and for all $t \in[0, \tau]$ it holds that $\boldsymbol{\xi}(t) \in D$ and $\dot{\boldsymbol{\xi}}(t)=\boldsymbol{\mu}^{D}-\boldsymbol{\nu}^{D} \boldsymbol{\xi}(t)$. For initial values $\boldsymbol{x}_{0} \in D$, there exists a function $\boldsymbol{\xi}(t)$ and a $\tau(\tau>0)$, such that $\boldsymbol{\xi}(t)$ is the unique solution for (11) on [0, $\tau]$.

Let $\phi_{i}$ be a function from $\mathcal{D}_{r}$ to $\Omega_{i}$, defined as

$$
\phi_{i}(D)=\mu_{i}^{D} / \nu_{i}^{D}
$$

It follows directly from (11) that all solutions $\boldsymbol{\xi}(t)$ in $D$ monotonically converge towards a so-called target equilibrium $\boldsymbol{x}=\boldsymbol{\phi}(D)$, with

$$
\phi(D)=\left(\phi_{1}(D), \ldots, \phi_{n}(D)\right)^{\prime} .
$$

Intuitively speaking, the target equilibrium level $\phi_{i}(D)$ of $x_{i}$ gives an indication of the strength of gene expression in the regulatory domain. If $\phi(D) \in D$, then for $t \rightarrow \infty$ all solutions in $D$ approach the target equilibrium, which is then a stable equilibrium

point of the system, also called regular steady state [61]. If $\phi(D) \notin D$, all solutions will leave $D$ at some point.

In the example of Figure 2, as can be easily checked from the state equations, we 
have $M_{a}=\left\{0, \kappa_{a}\right\}, N_{a}=\left\{\gamma_{a}\right\}$ for protein A, and $M_{b}=\left\{0, \kappa_{b}\right\}, N_{b}=\left\{\gamma_{b}\right\}$ for protein B. In the regulatory domain $D^{1}$ in Figure $3(\mathrm{~b})$, the state equations simplify to

$$
\begin{gathered}
\dot{x}_{a}=\kappa_{a}-\gamma_{a} x_{a}, \\
\dot{x}_{b}=\kappa_{b}-\gamma_{b} x_{b} .
\end{gathered}
$$

As a consequence, the target equilibrium $\phi\left(D^{1}\right)$ of $D^{1}$ equals $\left(\kappa_{a} / \gamma_{a}, \kappa_{b} / \gamma_{b}\right)^{\prime}$, which lies outside $D^{1}$. The trajectories in $D^{1}$ will therefore leave the domain at some point. Different regulatory domains generally have different target equilibria. For instance, in the regulatory domain $D^{3}$ defined by $0 \leq x_{a}<\theta_{a}^{1}$ and $\theta_{b}^{1}<x_{b}<\theta_{b}^{2}$, the target equilibrium is given by $\left(0, \kappa_{b} / \gamma_{b}\right)^{\prime}$.

\subsection{Analysis in switching domains}

In the switching domains of $\Omega,(2)$ is generally not defined, because one or more of the concentration variables take a threshold value. This is not much of a problem, if solution trajectories in one regulatory domain arrive at a switching domain from which they can be continued in another regulatory domain $[16,60]$. The trajectories arriving at $D^{2}$ from $D^{1}$ in Figure 4(a) are a case in point. However, if solution trajectories in different regulatory domains evolve towards the same switching domain, as is the case for trajectories arriving at $D^{4}$ from $D^{3}$ or $D^{5}$ (Figure 4(b)), mathematical perplexities arise. In the framework of the previous subsection, there is no indication on how the solutions in $D^{3}$ and $D^{5}$ can be continued. ${ }^{\ddagger}$
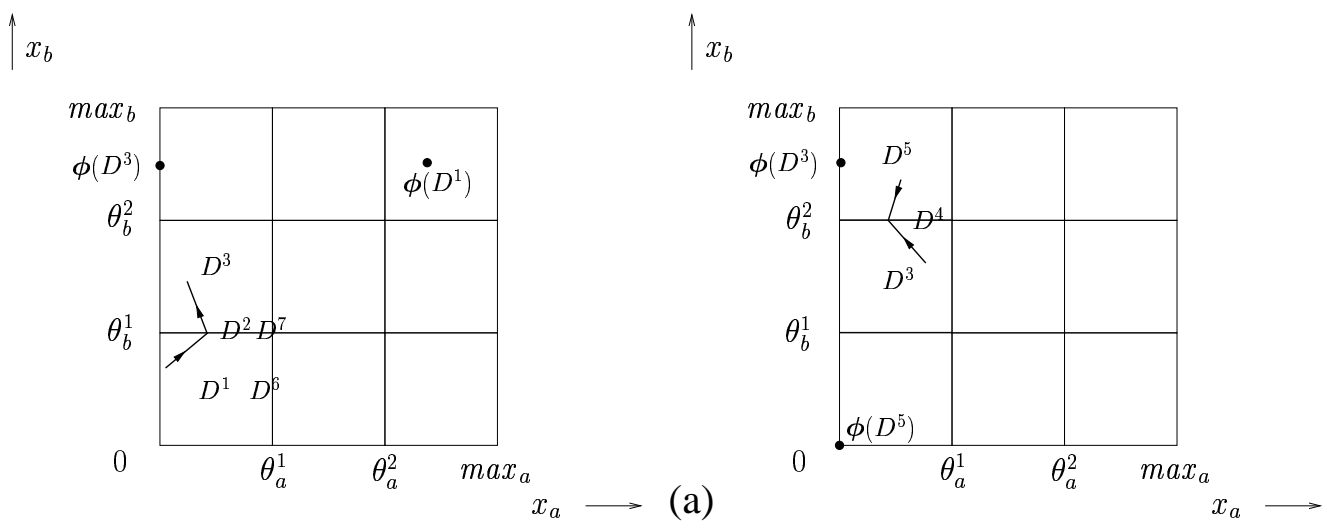

Figure 4: Examples of the behavior of the system of Figure 2 at threshold boundaries. The figures show the regulatory domains $D^{1}, \ldots, D^{5}$, and the target equilibria $\phi\left(D^{1}\right), \phi\left(D^{3}\right), \phi\left(D^{5}\right)$. In (a) the solution trajectories in $D^{1}$ can be continued in $D^{3}$, whereas in (b) the solution trajectories in $D^{3}$ cannot be continued in $D^{5}$.

The underlying cause of this problem is the occurrence of discontinuities in the right-hand side of (2), due to the use of step functions. The discontinuities occur at

${ }^{\ddagger}$ In $[47,52], D^{2}$ is called a transparent wall and $D^{4}$ a black wall. Another problem, not shown here, occurs when a switching domain is a white wall, that is, when the trajectories in two regulatory domains evolve from a common bounding switching domain. 
threshold hyperplanes, which separate regulatory domains in which the dynamics of the regulatory network is described by a different system of differential equations (11). In order to deal with these discontinuities in a general and rigorous way, we will use a method originally proposed by Filippov [19]. This method, recently applied by Gouzé and Sari [31] to PL systems of the form (2), consists of extending a system of differential equations with discontinuous right-hand sides into a system of differential inclusions. By means of this extension we can explicitly describe the behavior of the system in a threshold hyperplane.

More precisely, the differential equations (2) are extended into differential inclusions

$$
\dot{\boldsymbol{x}} \in \boldsymbol{H}(\boldsymbol{x}),
$$

where $\boldsymbol{H}: \Omega \rightarrow 2^{\Omega}$ is a set-valued function. ${ }^{\S}$ For $\boldsymbol{x} \in D$, and $D$ a regulatory domain, we define $\boldsymbol{H}(\boldsymbol{x})$ simply as

$$
\boldsymbol{H}(\boldsymbol{x})=\left\{\boldsymbol{\mu}^{D}-\boldsymbol{\nu}^{D} \boldsymbol{x}\right\} .
$$

Notice that the extension of the PL system agrees with the original system in the regulatory domains. If $D$ is a switching domain, $\boldsymbol{H}(\boldsymbol{x})$ is defined by

$$
\boldsymbol{H}(\boldsymbol{x})=\overline{c o}\left(\left\{\boldsymbol{\mu}^{D^{\prime}}-\boldsymbol{\nu}^{D^{\prime}} \boldsymbol{x} \mid D^{\prime} \in R(D)\right\}\right) .
$$

The smallest closed convex set $\overline{c o}(E)$ of a set $E$ is the intersection of all closed convex sets containing $E$ [19]. In the case of switching domains, $\boldsymbol{H}(\boldsymbol{x})$ will not generally be single-valued.

An absolutely continuous function $\boldsymbol{\xi}\left(t, \boldsymbol{x}_{0}, \boldsymbol{\theta}, \boldsymbol{\kappa}, \boldsymbol{\gamma}\right)$ is a solution of (14) in the sense of Filippov on $[0, \tau](\tau>0)$, if $\boldsymbol{\xi}(0)=\boldsymbol{x}_{0}$ and for almost all $t \in[0, \tau]$ it holds that $\dot{\boldsymbol{\xi}}(t) \in \boldsymbol{H}(\boldsymbol{\xi}(t))$ [19]. The qualification 'for almost all $t \in[0, \tau]$ ' means that the set of time-points for which the condition does not hold is of measure 0 . In particular, the condition is not satisfied at time-points when the solution arrives at or leaves a switching domain $D$. If no misunderstanding is possible, we will often simply speak of 'a solution of (14),' instead of 'a solution of (14) in the sense of Filippov.' For all initial values $\boldsymbol{x}_{0} \in \Omega$ there exists a solution of (14) on some $[0, \tau]$ [19]. However, this solution is not guaranteed to be unique, due to the generalization of the differential equations to differential inclusions.

In order to get an intuitive feeling for the meaning of the above concepts, consider again the examples in Figure 4. In the first case, $D^{2}$ is the switching domain bounding the regulatory domains $D^{1}$ and $D^{3}$. If $\boldsymbol{x} \in D^{2}$, then $\boldsymbol{H}(\boldsymbol{x})$ is the smallest closed convex set including the end-points of the vectors $\boldsymbol{\mu}^{D^{1}}-\boldsymbol{\nu}^{D^{1}} \boldsymbol{x}$ and $\boldsymbol{\mu}^{D^{3}}-\boldsymbol{\nu}^{D^{3}} \boldsymbol{x}$ starting at $\boldsymbol{x}$. This set is graphically represented in Figure 5(a) by the linear segment connecting the end-points of the vectors. An absolutely continuous function $\boldsymbol{\xi}(t)$ defined on a time-interval $[0, \tau]$, which remains in $D^{1}$ and satisfies (14) for $t \in[0, \sigma[$, crosses $\boldsymbol{x} \in D^{2}$ at $t=\sigma$, and remains in $D^{3}$ and satisfies (14) on $\left.\left.t \in\right] \sigma, \tau\right], \sigma<\tau$, is a

$\S_{2} 2^{E}$ represents the powerset (the set of subsets) of the set $E$. 

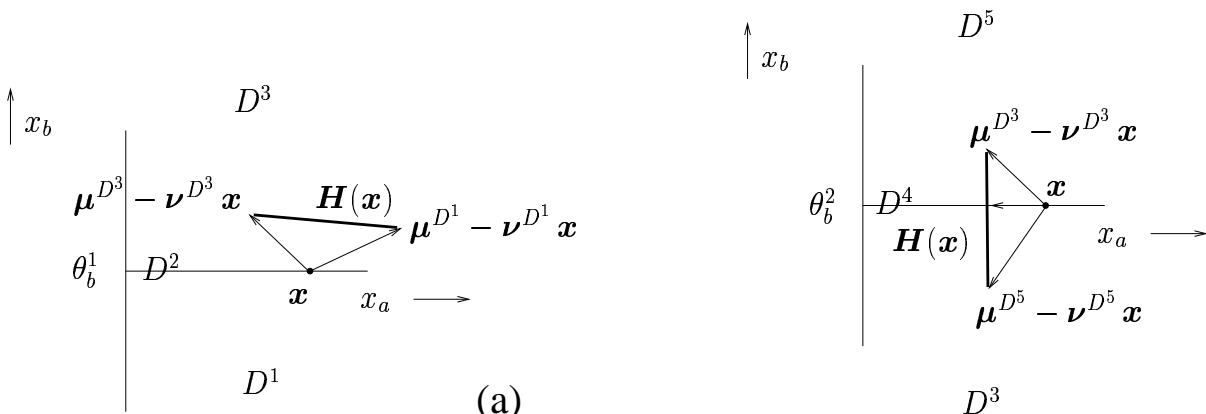

Figure 5: Behavior of the system of Figure 1 at a point $\boldsymbol{x}$ in a threshold boundary, when the differential equations are generalized into differential inclusions by the method of Filippov. Whereas in (a) solution trajectories cross $D^{2}$ instantaneously, in (b) they slide along $D^{4}$.

solution. In the second example of Figure $4, \boldsymbol{H}(\boldsymbol{x})$ is a linear segment connecting the end-points of the vectors $\boldsymbol{\mu}^{D^{3}}-\boldsymbol{\nu}^{D^{3}} \boldsymbol{x}$ and $\boldsymbol{\mu}^{D^{5}}-\boldsymbol{\nu}^{D^{5}} \boldsymbol{x}$. Contrary to the previous example, $\boldsymbol{H}(\boldsymbol{x})$ intersects with the threshold boundary, because the vector fields in $D^{3}$ and $D^{5}$ are directed towards $D^{4}$ (Figure 5(b)). An absolutely continuous function $\boldsymbol{\xi}(t)$ defined on a time-interval $[0, \tau]$, which remains in $D^{3}$ and satisfies (14) for $t \in[0, \sigma$, arrives at $\boldsymbol{x} \in D^{4}$ at $t=\sigma$, and slides along $D^{4}$ and satisfies (14) on $\left.\left.t \in\right] \sigma, \tau\right], \sigma<\tau$, is a solution.

For every domain $D$, a target equilibrium set $\Phi(D)$ can be defined. If $D$ is a regulatory domain, then

$$
\Phi(D)=\{\phi(D)\}
$$

where $\phi(D)$ is the target equilibrium of $D$, defined by (13). If $D$ is a switching domain, the definition is a little bit more complicated. Let $D$ be a switching domain of order $k$, contained in the $(n-k)$-dimensional hyperplane $C$. Then

$$
\Phi(D)=C \cap \overline{c o}\left(\left\{\phi\left(D^{\prime}\right) \mid D^{\prime} \in R(D)\right\}\right) .
$$

That is, $\Phi(D)$ is the smallest closed convex set of the target equilibria of regulatory domains $D^{\prime}$ having $D$ in their boundary, intersected with the threshold hyperplane containing $D$. In the case of switching domains, $\Phi(D)$ is not generally a single point.

Gouzé and Sari [31] have shown that all solutions $\boldsymbol{\xi}(t)$ either cross a domain $D$ instantaneously or remain in it on some extended time-interval, while converging towards $\Phi(D)$. For the latter solutions to exist, it must hold

$$
\Phi(D) \neq\{\} .
$$

In regulatory domains, (19) is always satisfied and solutions monotonically converge towards $\Phi(D)$, as discussed in Section 3.2. In switching domains, if (19) is satisfied, the convergence of the solutions in $D$ is monotonic in a weak sense (Appendix A). ${ }^{9}$

T The solutions satisfying (19) in switching domains are known as sliding mode solutions in control theory [13]. 
If $\Phi(D) \cap D=\{\}$ all solutions will leave $D$ at some point. On the other hand, if $\Phi(D) \cap D \neq\{\}$, there exist solutions in $D$ that approach or enter the target equilibrium set $\Phi(D)$ as $t \rightarrow \infty$. Every $\phi \in \Phi(D) \cap D$ is an equilibrium point of the system, in the sense that there exists a solution $\boldsymbol{\xi}\left(t, \boldsymbol{x}_{0}, \boldsymbol{\theta}, \boldsymbol{\kappa}, \boldsymbol{\gamma}\right)$, such that $\boldsymbol{\xi}(t)=\boldsymbol{\phi}$ for all $t \geq 0$. In the special case that $\Phi(D)$ is a singleton, the equilibrium point $\phi$ has been called a singular steady state [61] of the system. Whether this equilibrium point is stable or unstable must be determined through further analysis.

Consider the examples in Figure 6. The target equilibrium set $\Phi\left(D^{2}\right)$ of the switching domain $D^{2}$ is defined, following (18), by the intersection of $\overline{c o}\left(\left\{\phi\left(D^{1}\right), \phi\left(D^{3}\right)\right\}\right)$ and the threshold boundary $x_{b}=\theta_{b}^{1}$. The smallest closed convex set consists of the linear segment connecting the points $\left(\kappa_{a} / \gamma_{a}, \kappa_{b} / \gamma_{b}\right)^{\prime}$ and $\left(0, \kappa_{b} / \gamma_{b}\right)^{\prime}$, as shown in (a). $\overline{c o}\left(\left\{\phi\left(D^{1}\right), \phi\left(D^{3}\right)\right\}\right)$ and the threshold boundary $x_{b}=\theta_{b}^{1}$ do not intersect in the figure, so $\Phi\left(D^{2}\right)=\{\}$. Following criterion (19), there are no solutions remaining in $D^{2}$. This is different in the case of $D^{4}$. Here, the target equilibrium set $\Phi\left(D^{4}\right)$ is given by the intersection of $\overline{c o}\left(\left\{\phi\left(D^{3}\right), \phi\left(D^{5}\right)\right\}\right)$, the linear segment connecting the points $\left(0, \kappa_{b} / \gamma_{b}\right)^{\prime}$ and $(0,0)^{\prime}$, and the threshold boundary $x_{b}=\theta_{b}^{2}$. Consequently, $\Phi\left(D^{4}\right)$ equals $\left\{\left(0, \theta_{b}^{2}\right)^{\prime}\right\}$, and there exists a solution remaining in $D^{4}$, converging towards $\left(0, \theta_{b}^{2}\right)$. Because the target equilibrium lies inside $D^{4}$, it is an equilibrium point of the system. Closer analysis reveals that it is stable.
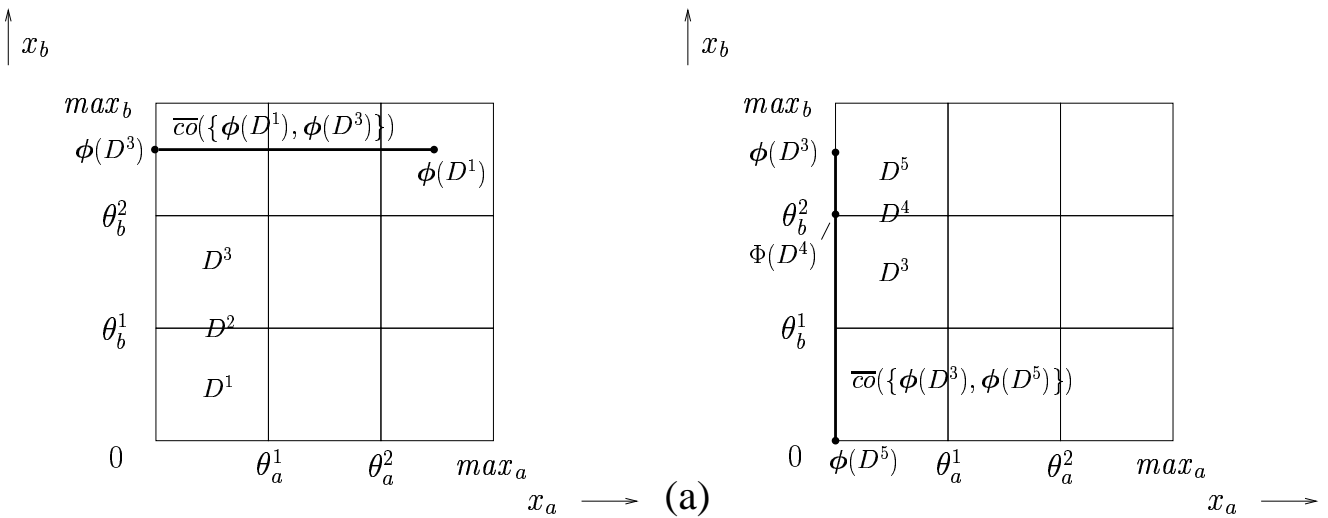

Figure 6: Determination of the target equilibrium sets (a) $\Phi\left(D^{2}\right)=\{\}$ and (b) $\Phi\left(D^{4}\right)=\left\{\left(0, \theta_{b}^{2}\right)^{\prime}\right\}$.

\section{Qualitative description of dynamics of piecewise-linear models}

The mathematical framework presented in the previous section suggests an intuitive qualitative description of the dynamics of regulatory systems described by the PL models (2). This description is based on an abstraction of the state of a regulatory system, a qualitative state, consisting of the domain $D$ in which the system resides and the position of the target equilibrium set $\Phi(D)$ with respect to $D$. There exists a transition between two qualitative states $Q S$ and $Q S^{\prime}$, corresponding to contiguous domains $D$ and $D^{\prime}$, if some solution trajectories starting in $D$ reach $D^{\prime}$, without passing 
through an intermediate domain. The sets of qualitative states and transitions between qualitative states define a state transition graph concisely representing the qualitative dynamics of the regulatory system. In this section we will elaborate the ideas of qualitative state, transition between qualitative states, and state transition graph.

\subsection{Qualitative states}

As a preliminary step, we define a function $v: \mathcal{D} \times \Omega \rightarrow\{-1,0,1\}^{n}$ that maps a domain $D$ and a point $\boldsymbol{e}$ to a sign vector describing the relative position of $D$ and $\boldsymbol{e}$. More precisely, we define $v(D, \boldsymbol{e})$, such that the $i$ th component of the sign vector, $1 \leq i \leq n$, is given by

$$
v(D, \boldsymbol{e})_{i}=\left\{\begin{aligned}
1 & , \text { if for all } d \in D_{i}: e_{i}>d \\
0 & , \text { if for some } d \in D_{i}: e_{i}=d \\
-1 & , \text { if for all } d \in D_{i}: e_{i}<d
\end{aligned}\right.
$$

As an example, consider the relative position of domains and their target equilibria in Figure 4. We find $v\left(D^{1}, \phi\left(D^{1}\right)\right)=(1,1), v\left(D^{3}, \phi\left(D^{3}\right)\right)=(0,1)$, and $v\left(D^{5}, \phi\left(D^{5}\right)\right)=$ $(0,-1)$.

The definition of $v$ can be generalized to obtain a set function $V: \mathcal{D} \times 2^{\Omega} \rightarrow$ $2^{\{-1,0,1\}^{n}}$, which maps a domain $D$ and a set $E$ to a set of sign vectors describing the relative position of $D$ and points in $E$ :

$$
V(D, E)=\{v(D, \boldsymbol{e}) \mid \boldsymbol{e} \in E\} .
$$

For example, in Figure 7, we have $V\left(D^{1}, \Phi\left(D^{1}\right)\right)=\{(1,1)\}, V\left(D^{2}, \Phi\left(D^{2}\right)\right)=$ \{\}$, V\left(D^{3}, \Phi\left(D^{3}\right)\right)=\{(0,1)\}, V\left(D^{4}, \Phi\left(D^{4}\right)\right)=\{(0,0)\}$, and $V\left(D^{5}, \Phi\left(D^{5}\right)\right)=$ $\{(0,-1)\}$. Moreover, $V\left(D^{1}, D^{2}\right)=\{(0,1)\}, V\left(D^{1}, D^{7}\right)=\{(1,1)\}$, and $V\left(D^{1}, D^{2} \cup\right.$ $\left.D^{7}\right)=\{(0,1),(1,1)\}$.

To every domain $D \in \mathcal{D}$, we associate a qualitative state $Q S$ defined as follows:

$$
Q S=\langle D, V(D, \Phi(D))\rangle .
$$

That is, the qualitative state describes the relative position of $D$ and its target equilibrium set $\Phi(D)$, thus capturing the local dynamics of the system in $D$. The set of qualitative states associated with the domains $\mathcal{D}$ is denoted by $\mathcal{Q S}$. In the example system we have $Q S^{1}=\left\langle D^{1},\{(1,1)\}\right\rangle, Q S^{2}=\left\langle D^{2},\{\}\right\rangle, Q S^{3}=\left\langle D^{3},\{(0,1)\}\right\rangle$, $Q S^{4}=\left\langle D^{4},\{(0,0)\}\right\rangle$, and $Q S^{5}=\left\langle D^{5},\{(0,-1)\}\right\rangle$ (Figure 7).

\subsection{Transitions between qualitative states}

Let $Q S, Q S^{\prime} \in \mathcal{Q S}$ be two qualitative states associated with contiguous domains $D, D^{\prime} \in \mathcal{D}$. Contiguity implies that either $D^{\prime}$ is in the boundary of $D$, or $D$ is in the boundary of $D^{\prime}$. Consider $D^{\prime} \in A(D)$. There exists a transition $T=\left\langle Q S, Q S^{\prime}\right\rangle$ between the qualitative states, if there is a solution $\boldsymbol{\xi}\left(t, \boldsymbol{x}_{0}, \boldsymbol{\theta}, \boldsymbol{\kappa}, \boldsymbol{\gamma}\right)$ defined on a finite time interval $[0, \tau]$, such that 


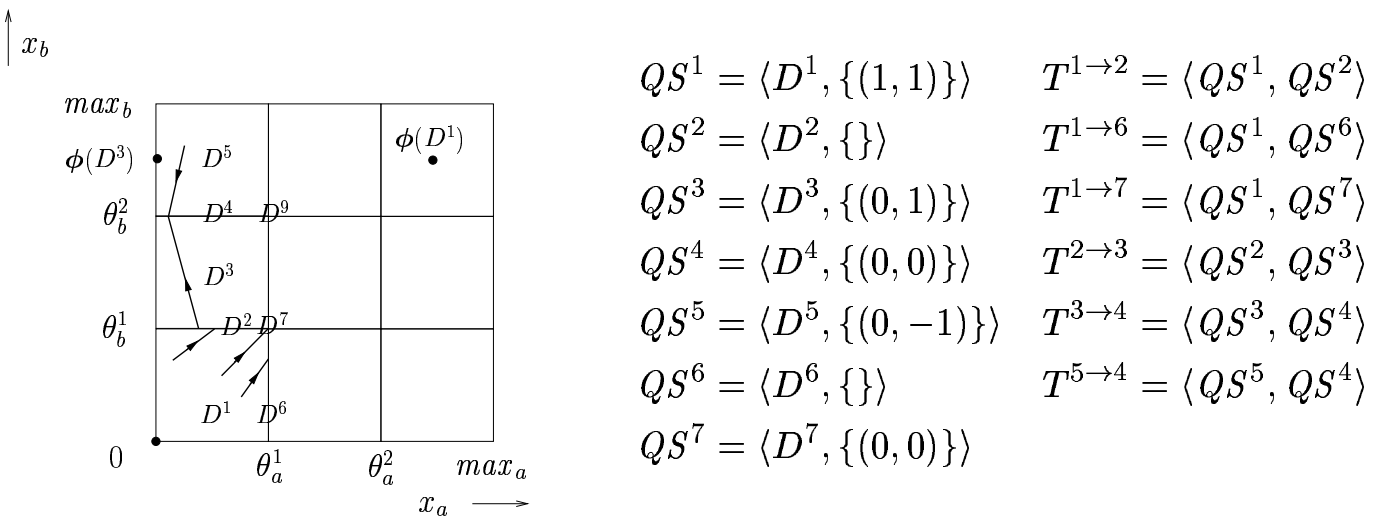

Figure 7: Examples of qualitative states and transitions between qualitative states for the PL model in Figure 2.

1. $\boldsymbol{\xi}(t) \in D$ for $0 \leq t<\tau$, and

2. $\boldsymbol{\xi}(\tau) \in D^{\prime}$.

The definition expresses that there exists a solution trajectory reaching $D^{\prime}$ from $D$ in finite time, without passing through a third domain. A similar definition covers the case that $D \in A\left(D^{\prime}\right)$. For a transition $T=\left\langle Q S, Q S^{\prime}\right\rangle$ to exist, there must be a solution $\boldsymbol{\xi}\left(t, \boldsymbol{x}_{0}, \boldsymbol{\theta}, \boldsymbol{\kappa}, \boldsymbol{\gamma}\right)$ defined on a finite time interval $[0, \tau]$, such that

1. $\boldsymbol{\xi}(0) \in D$, and

2. $\boldsymbol{\xi}(t) \in D^{\prime}$ for $0<t \leq \tau$.

We will use $\mathcal{T}$ to denote the set of transitions between the qualitative states $\mathcal{Q S}$ of a regulatory system.

Figure 7 gives a few examples of transitions between qualitative states in the example system. For the parameter values in Figure 2, trajectories starting in $D^{1}$ converge towards $\Phi\left(D^{1}\right)$ and reach the boundary domains $D^{2}, D^{6}$, or $D^{7}$ in finite time, thus giving rise to transitions $T^{1 \rightarrow 2}=\left\langle Q S^{1}, Q S^{2}\right\rangle, T^{1 \rightarrow 6}=\left\langle Q S^{1}, Q S^{6}\right\rangle$, and $T^{1 \rightarrow 7}=$ $\left\langle Q S^{1}, Q S^{7}\right\rangle$, respectively. Solution trajectories starting in $D^{2}$ will immediately enter $D^{3}, D^{2} \in A\left(D^{3}\right)$, so that $T^{2 \rightarrow 3}=\left\langle Q S^{2}, Q S^{3}\right\rangle$ is a transition of the system. All trajectories in $D^{3}$ reach $D^{4} \in A\left(D^{3}\right)$ in finite time, which results in the transition $T^{3 \rightarrow 4}=\left\langle Q S^{3}, Q S^{4}\right\rangle$. There is no transition possible from $D^{4}$, because all trajectories starting in $D^{4}$ remain in this domain, sliding towards the target equilibrium set $\Phi\left(D^{4}\right)$ in $D^{4}$. In other words, no solutions reach $D^{9}$, domain in the boundary of $D^{4}$, and no solutions reach $D^{3}$ and $D^{5}$, domains having $D^{4}$ in their boundary.

What would happen if we choose the parameter values such that $\phi\left(D^{3}\right)$ is located in the threshold boundary defined by $x_{b}=\theta_{b}^{2}$ ? The trajectories starting in $D^{3}$ would still reach $D^{4}$, but not in finite time! So, according to the definition, there would be no transition between $Q S^{3}$ and $Q S^{4}$. This akward situation can be avoided under the condition that, if there exists $\phi \in \Phi(D)$ included in the boundary of $D$, i.e. $\phi \in \Phi(D) \cap B(D)$, then there exists another $\boldsymbol{\phi}^{\prime} \in \Phi(D)$ not included in the boundary 
of $D$, i.e. $\phi^{\prime} \notin \Phi(D) \cap B(D)$, such that $v(D, \phi)=v\left(D, \phi^{\prime}\right)$. The condition guarantees that, if there are solutions reaching the boundary in infinite time, there are also solutions doing so in finite time. For regulatory domains, this simplifies to the generic assumption that $\phi(D)$ is not located in the boundary of $D$.

\subsection{State transition graph}

A state transition graph is a directed graph $G$ consisting of the qualitative states $\mathcal{Q S}$ of a system and the transitions $\mathcal{T}$ between these qualitative states, that is, $G=\langle\mathcal{Q S}, \mathcal{T}\rangle$. Figure 8 shows the state transition graph obtained for the model in Figure 2. The state transition graph obtained from a PL model provides a qualitative picture of the dynamics of a genetic regulatory system, as will be discussed below.
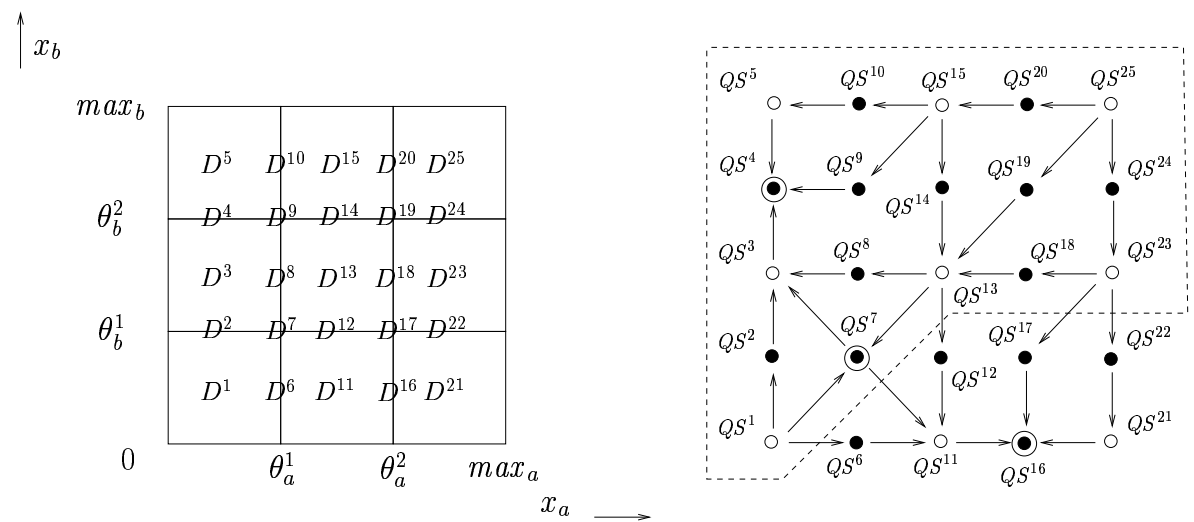

Figure 8: Phase space and state transition graph for the PL model in Figure 2. Qualitative states associated with regulatory and switching domains are indicated by unfilled and filled dots, respectively. Qualitative equilibrium states are circled in addition. The attraction set of the qualitative equilibrium state $Q S^{4}$ is marked by the dotted border.

Proposition 4.1: Let $Q S=\langle D, V(D, \Phi(D))\rangle$ be a qualitative state. There exist solutions remaining in $D$, iff $V(D, \Phi(D)) \neq\{\}$. Conversely, no such solutions exist, iff $V(D, \Phi(D))=\{\}$.

Proof: There exist solutions remaining in $D$, iff $D$ is a regulatory domain or $D$ is a switching domain satisfying condition (19). In both cases, $\Phi(D) \neq\{\}$ and hence $V(D, \Phi(D)) \neq\{\}$. There do not exist any such solutions, iff $D$ is a switching domain not satisfying condition (19). In that case, $\Phi(D)=\{\}$ and hence $V(D, \Phi(D))=\{\}$.

A qualitative state $Q S=\langle D, V(D, \Phi(D))\rangle$, such that $V(D, \Phi(D)) \neq\{\}$, will be called persistent. On the other hand, a qualitative state for which $V(D, \Phi(D))=\{\}$ will be called instantaneous. The instantaneous qualitatives states in Figure 8 are $Q S^{2}$, $Q S^{6}, Q S^{8}, Q S^{9}, Q S^{10}, Q S^{12}, Q S^{14}, Q S^{17}, Q S^{18}, Q S^{19}, Q S^{20}, Q S^{22}$, and $Q S^{24}$. Instantaneous qualitative states can be removed from a state transition graph, because 
their biological significance is limited. This results in a state transition graph that is smaller and hence easier to interpret.

Proposition 4.2: Let $Q S=\langle D, V(D, \Phi(D))\rangle$ be a qualitative state. $D$ contains an equilibrium point, iff $\mathbf{0} \in V(D, \Phi(D))$.

Proof: If $D$ is a regulatory domain, then $\Phi(D)=\{\phi(D)\} \cdot \phi(D)$ is an equilibrium point, iff $\phi(D) \in D$, that is, iff $v(D, \phi(D))=\mathbf{0}$. If $D$ is a switching domain, then $\Phi(D)=C \cap \overline{c o}\left(\left\{\phi\left(D^{\prime}\right) \mid D^{\prime} \in R(D)\right\}\right) . \phi \in \Phi(D)$ is an equilibrium point, iff $\phi \in D$, that is, iff $v(D, \phi)=\mathbf{0}$.

A qualitative state such that $0 \in V(D, \Phi(D))$ will be called a qualitative equilibrium state. Examples of qualitative equilibrium states in the state transition graph of Figure 8 are $Q S^{4}=\left\langle D^{4},\{(0,0)\}\right\rangle, Q S^{7}=\left\langle D^{7},\{(0,0)\}\right\rangle$, and $Q S^{16}=\left\langle D^{16},\{(0,0)\}\right\rangle$. The qualitative equilibrium states $Q S^{4}$ and $Q S^{16}$ correspond to stable equilibria of the system, located at $\left(0, \theta_{b}^{2}\right)^{\prime}$ and $\left(\theta_{a}^{2}, 0\right)^{\prime}$, respectively. The qualitative equilibrium state $Q S^{7}$ corresponds to an unstable equilibrium located at $\left(\theta_{a}^{1}, \theta_{b}^{1}\right)^{\prime}$. The stable equilibria present the two functional states of the system: (1) gene $a$ on and gene $b$ off, (2) gene $a$ off and gene $b$ on. This confirms the results of earlier mathematical studies of genetic regulatory networks with the same or a similar structure (e.g., [7, 30, 35, 36, 66, 70]).

The qualitative states from which a qualitative equilibrium state is reachable together form the attraction set of that state. Figure 8 shows the attraction set of the qualitative equilibrium state $Q S^{4}$. Notice that a qualitative state may be a member of several attraction sets, like $Q S^{1}$ in Figure 8, which is in the attraction sets of all three qualitative equilibrium states.
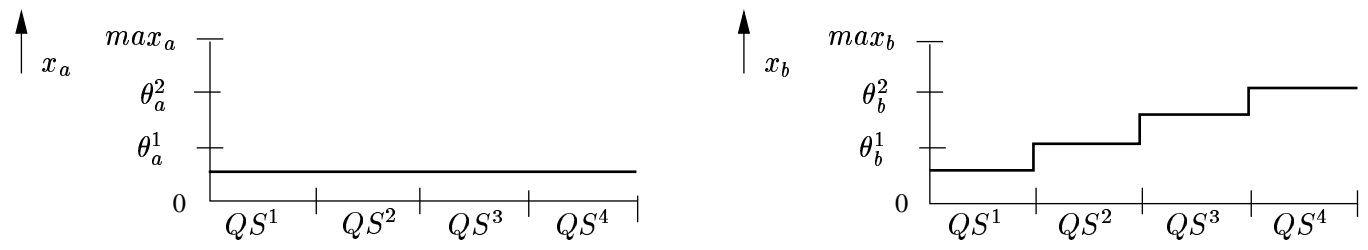

Figure 9: Detailed description of the qualitative behavior $\left(Q S^{1}, Q S^{2}, Q S^{3}, Q S^{4}\right)$ in Figure 8.

A path in the state transition graph will be called a qualitative behavior of the system. For instance, $\left(Q S^{1}, Q S^{2}, Q S^{3}, Q S^{4}\right),\left(Q S^{1}, Q S^{6}, Q S^{11}, Q S^{16}\right)$, and $\left(Q S^{1}, Q S^{7}\right)$ are qualitative behaviors of the system. Each of these behaviors leads from $Q S^{1}$ to one of the qualitative equilibrium states. The qualitative behaviors in a state transition graph describe how the bounds on protein concentrations evolve over time, according to the sequence of transitions between qualitative states. In Figure 9, one of the qualitative behaviors of the example system is explored in more detail. A cyclic qualitative behavior will be called a qualitative cycle. Like for qualitative equilibrium states, we can define an attraction set for qualitative cycles. The relation between limit cycles 
and qualitative cycles has been studied for some special cases $[14,28,29,48,60]$. Figure 8 does not contain any qualitative cycles.

There exists an important relation between the qualitative behaviors in the state transition graph and the solutions of a PL model. Let $\boldsymbol{\xi}\left(t, \boldsymbol{x}_{0}, \boldsymbol{\theta}, \boldsymbol{\kappa}, \boldsymbol{\gamma}\right)$ be a solution of a PL model on a finite time-interval $[0, \tau]$. We assume that $\boldsymbol{x}_{0} \in \Omega$, so that $\boldsymbol{\xi}(t)$ remains in $\Omega$ on $[0, \tau]$ (Section 3.1).

PROPOSITION 4.3: If $\boldsymbol{\xi}(t)$ is a solution passing through the finite sequence of domains $\left(D^{0}, \ldots, D^{m}\right)$ on the finite time-interval $[0, \tau]$, then

$$
\left(Q S^{0}=\left\langle D^{0}, V\left(D^{0}, \Phi\left(D^{0}\right)\right)\right\rangle, \ldots, Q S^{m}=\left\langle D^{m}, V\left(D^{m}, \Phi\left(D^{m}\right)\right)\right\rangle\right)
$$

is a qualitative behavior in the state transition graph.

Proof: Because $\boldsymbol{\xi}(t)$ passes through the sequence of domains $\left(D^{0}, \ldots, D^{m}\right)$ on the finite time-interval $[0, \tau]$, there exist transitions between qualitative states $Q S^{k}$ and $Q S^{k+1}, 0 \leq k<m$. As a consequence, $\left(Q S^{0}, \ldots, Q S^{m}\right)$ is a qualitative behavior in the state transition graph.

Informally speaking, the proposition means that the set of solutions passing through a finite sequence of domains on a finite time-interval of the PL model is covered by the state transition graph. It allows us to infer that, if a qualitative behavior $\left(Q S^{0}, \ldots, Q S^{m}\right)$ is absent from the state transition graph, then there does not exist any solution of the PL model passing through the sequence of domains $\left(D^{0}, \ldots, D^{m}\right)$.

\section{Qualitative piecewise-linear models}

Most of the time, precise numerical values for the threshold and rate parameters in a PL model are not available. However, instead of specifying precise numerical values, it is often possible to supplement the state equations with inequality constraints on the parameter values. The inequality constraints express weak, but reliable information on the regulatory interactions that can be inferred from biological data. The resulting, so-called qualitative PL model subsumes a set of quantitative PL models, the qualitative dynamics of each of which can be described by means of a state transition graph. In the next section, we will show that, under some conditions to be spelled out, all quantitative PL models subsumed by a qualitative PL model yield the same state transition graph.

The first type of inequality constraints in a qualitative PL model are the so-called threshold inequalities. They are obtained by ordering the $p_{i}$ threshold concentrations of gene $i$, that is,

$$
0<\theta_{i}^{1}<\ldots<\theta_{i}^{p_{i}}<\max _{i} .
$$

In the case of protein A, there are two threshold concentrations: $\theta_{a}^{1}$ is the threshold for the repression of gene $b$, while $\theta_{a}^{2}$ is the threshold for the repression of gene $a$. 
State equation for gene $a$ : $\dot{x}_{a}=\kappa_{a} s^{-}\left(x_{a}, \theta_{a}^{2}\right) s^{-}\left(x_{b}, \theta_{b}^{1}\right)-\gamma_{a} x_{a}$

Threshold inequalities: $0<\theta_{a}^{1}<\theta_{a}^{2}<\max _{a}$

Equilibrium inequalities: $\theta_{a}^{2}<\frac{\kappa_{a}}{\gamma_{a}}<\max _{a}$

State equation for gene $b$ : $\dot{x}_{b}=\kappa_{b} s^{-}\left(x_{a}, \theta_{a}^{1}\right) s^{-}\left(x_{b}, \theta_{b}^{2}\right)-\gamma_{b} x_{b}$

Threshold inequalities: $0<\theta_{b}^{1}<\theta_{b}^{2}<\max _{b}$

Equilibrium inequalities: $\theta_{b}^{2}<\frac{\kappa_{b}}{\gamma_{b}}<\max _{b}$

Figure 10: State equations, threshold inequalities, and equilibrium inequalities forming the qualitative PL model of the network of Figure 1.

Assuming the first to be lower than the second, we obtain the threshold inequalities $0<\theta_{a}^{1}<\theta_{a}^{2}<\max _{a}$. The ordering of the thresholds of protein B can be determined likewise, giving rise to $0<\theta_{b}^{1}<\theta_{b}^{2}<\max _{b}$, with $\theta_{b}^{1}$ being the threshold for gene $a$ repression and $\theta_{b}^{2}$ the threshold for gene $b$ autorepression (Figure 10).

Second, the possible target equilibrium levels of $x_{i}$ in different regulatory domains $D \in \mathcal{D}_{r}$ can be ordered with respect to the threshold concentrations. The resulting equilibrium inequalities define the strength of gene expression in the domain in a qualitative way, on the scale of ordered threshold concentrations. More precisely, for every $\mu_{i} \in M_{i}, \nu_{i} \in N_{i}$, and $\mu_{i}, \nu_{i} \neq 0$, we specify one of the following pairs of inequalities:

$$
\begin{aligned}
& 0<\mu_{i} / \nu_{i}<\theta_{i}^{1}, \\
& \theta_{i}^{1}<\mu_{i} / \nu_{i}<\theta_{i}^{2}, \\
& \ldots \\
& \theta_{i}^{p_{i}}<\mu_{i} / \nu_{i}<\max _{i} .
\end{aligned}
$$

The equilibrium inequalities for the example model are shown in Figure 10. In the absence of protein $\mathrm{B}\left(s^{-}\left(x_{b}, \theta_{b}^{1}\right)=1\right)$, while protein $\mathrm{A}$ has not yet reached its highest level $\left(s^{-}\left(x_{a}, \theta_{a}^{2}\right)=1\right)$, gene $a$ is expressed at a rate $\kappa_{a}$. The corresponding target equilibrium value $\kappa_{a} / \gamma_{a}$ of $x_{a}$ must be above the second threshold $\theta_{a}^{2}$, otherwise the concentration of the protein would not be able to reach or maintain a level at which the observed negative autoregulation of gene $a$ occurs (i.e., $\theta_{a}^{2}<\kappa_{a} / \gamma_{a}<\max _{a}$ ). In a similar way, the target equilibrium value $\kappa_{b} / \gamma_{b}$ is positioned above $\theta_{b}^{2}$, again to 
ensure that the negative autoregulation of gene $b$ at high concentrations of protein $\mathrm{B}$ can occur.

\section{Qualitative simulation}

In order to study the qualitative dynamics of a genetic regulatory system, we would like to know the possible state transition graphs for all quantitative PL models subsumed by the qualitative PL model. This requires the computation of qualitative states and transitions between qualitative states, which is a difficult problem in general, because $\Phi(D)$ may be a complex polyhedron in $\Omega$. In this section, we therefore propose a hyperrectangular overapproximation of $\Phi(D)$. The approximation has the property that in the region of the parameter space defined by the inequalities in the qualitative PL model, all quantitative PL models yield the same state transition graph. We will derive rules to efficiently compute this graph from the inequality constraints by symbolic instead of numerical means.

A state transition graph may become exceedingly large, as the number of domains, and hence qualitative states, grows exponentially with the dimension of the system (Section 3.1). For many purposes, it is sufficient to know which qualitative states are reachable from a given initial qualitative state, that is, which qualitative behaviors the system can exhibit when initially being in this state. We outline an algorithm for what will be called qualitative simulation, the generation of the reachable part of a state transition graph from a qualitative PL model and an initial domain.

\subsection{Computation of qualitative states}

In order to determine the qualitative state associated with a domain $D$, we need to compute $V(D, \Phi(D))$. Because $\Phi(D)$ may be a complex polyhedron in $\Omega$, we will compute $V(D, \Psi(D))$ instead of $V(D, \Phi(D))$, where $\Psi(D) \subseteq \Omega$ is the smallest closed hyperrectangle including $\Phi(D)$. Like $\Phi(D), \Psi(D)$ is a convex set, but it has a much simpler shape. More specifically, if $D$ is a regulatory domain, then $\Psi(D)=\Phi(D)=\{\phi(D)\}$. For a switching domain $D$ of order $k$ we have

$$
\Psi(D)=C \cap \overline{r e c t}\left(\left\{\boldsymbol{\phi}\left(D^{\prime}\right) \mid D^{\prime} \in R(D)\right\},\right.
$$

where $C$ is the $(n-k)$-dimensional threshold hyperplane containing $D$. The set $\overline{r e c t}(E)$ denotes the smallest closed hyperrectangle containing the set $E$. If $D$ is a switching domain, then in general $\Psi(D)$ will be an overapproximation of $\Phi(D)$. In Section 7 , we discuss the consequences of choosing this overapproximation.

The use of $V(D, \Psi(D))$ rather than $V(D, \Phi(D))$ much facilitates the computation of qualitative states, as demonstrated by the following rules.

Proposition 6.1: Let $D$ be a regulatory domain. $\boldsymbol{v} \in V(D, \Psi(D))$, iff for all $i$, $1 \leq i \leq n$, 


$$
v_{i}=\left\{\begin{aligned}
1 & , \text { if for all } d \in D_{i}: \phi_{i}(D)>d \\
0 & , \text { if for some } d \in D_{i}: \phi_{i}(D)=d \\
-1 & , \text { if for all } d \in D_{i}: \phi_{i}(D)<d
\end{aligned}\right.
$$

Proof: The result is a direct consequence of $\Psi(D)=\{\phi(D)\}$, for $D \in \mathcal{D}_{r}$, and the definition of $V(D, \Psi(D))$.

PROPOSITION 6.2: Let $D$ be a switching domain of order $k$ contained in the $(n-k)$ dimensional threshold hyperplane $C . V(D, \Psi(D)) \neq\{\}$, iff for all $i, 1 \leq i \leq n$, such that $x_{i}$ is a switching variable and $D_{i}=\{d\}$,

$$
\min _{D^{\prime} \in R(D)} \phi_{i}\left(D^{\prime}\right)<d<\max _{D^{\prime} \in R(D)} \phi_{i}\left(D^{\prime}\right)
$$

Proof: $V(D, \Psi(D)) \neq\{\}$, iff $\Psi(D) \neq\{\}$. For $D \in \mathcal{D}_{s}, \Psi(D)$ is defined by (25). $\Psi(D)$ can be decomposed into $\Psi_{1}(D) \times \ldots \times \Psi_{n}(D)$, because of its hyperrectangular shape. For every $i, 1 \leq i \leq n$,

$$
\Psi_{i}(D)=C_{i} \cap\left\{x_{i} \in \Omega_{i} \mid \min _{D^{\prime} \in R(D)} \phi_{i}\left(D^{\prime}\right) \leq x_{i} \leq \max _{D^{\prime} \in R(D)} \phi_{i}\left(D^{\prime}\right)\right\}
$$

If $x_{i}$ is a switching variable, then $C_{i}=D_{i}=\{d\}$, where $d$ equals some threshold $\theta_{i}^{k_{i}}$, $1 \leq k_{i} \leq p_{i}$. Condition (27) now follows with the observation that the inequalities are strict, because the models that interest us satisfy (24). As a consequence, $\phi_{i}\left(D^{\prime}\right)$ is not equal to a threshold.

Proposition 6.3: Let $D$ be a switching domain of order $k$ contained in the $(n-k)$ dimensional threshold hyperplane $C$, and $V(D, \Psi(D)) \neq\{\} \cdot \boldsymbol{v} \in V(D, \Psi(D))$, iff for all $i, 1 \leq i \leq n$, if $x_{i}$ is a switching variable, then

$$
v_{i}=0,
$$

and, if $x_{i}$ is a non-switching variable, then

$$
v_{i}=\left\{\begin{aligned}
1 & , \text { if for all } d \in D_{i}: \max _{D^{\prime} \in R(D)} \phi_{i}\left(D^{\prime}\right)>d \\
0 & , \text { if for some } d \in D_{i}: \min _{D^{\prime} \in R(D)} \phi_{i}\left(D^{\prime}\right) \leq d \leq \max _{D^{\prime} \in R(D)} \phi_{i}\left(D^{\prime}\right), \\
-1 & , \text { if for all } d \in D_{i}: \min _{D^{\prime} \in R(D)} \phi_{i}\left(D^{\prime}\right)<d
\end{aligned}\right.
$$

Proof: $\Psi(D)$ can be decomposed into $\Psi_{1}(D) \times \ldots \times \Psi_{n}(D)$, as in the proof of the previous proposition. If $x_{i}$ is a switching variable, then $C_{i}=D_{i}=\{d\}$ and $v_{i}=0$ by definition of $V$. If $x_{i}$ is a non-switching variable, then $\Psi_{i}(D)$ is defined as in (28) and the conditions on $v_{i}$ follow from the definition of $V$. 
The approximation of $\Phi(D)$ by $\Psi(D)$ has an important consequence. In principle, for different combinations of parameter values $\boldsymbol{\theta}, \boldsymbol{\kappa}, \boldsymbol{\gamma}$ consistent with the inequality constraints in the qualitative PL model, we could obtain a different relative position of a domain and its target equilibrium, and hence a different qualitative state. However, under the approximation it can be shown that every domain $D \in \mathcal{D}$ is associated with a unique qualitative state. As will be illustrated in Section 7.2, this is not generally true for qualitative states $\langle D, V(D, \Phi(D))\rangle$.

Lemma 6.1: For all quantitative PL models subsumed by a qualitative PL model, every domain $D \in \mathcal{D}$ is associated with a unique qualitative state $\langle D, V(D, \Psi(D))\rangle$.

Proof: The qualitative state $\langle D, V(D, \Psi(D))\rangle$ associated with $D$ is determined by the order relations specified in Propositions 6.1-6.3. In particular, for every $i, 1 \leq$ $i \leq n$, we need to determine the ordering of the target equilibrium value $\phi_{i}(D)$ and $D_{i}$ (if $D$ is a regulatory domain), or the ordering of the target equilibrium values $\phi_{i}\left(D^{\prime}\right), D^{\prime} \in R(D)$, and $D_{i}$ (if $D$ is a switching domain). These order relations are uniquely determined by the threshold and equilibrium inequalities (23)-(24) specified in a qualitative PL model.

Given a qualitative PL model, Propositions 6.1-6.3 can be used to compute the qualitative state associated with a domain, as suggested in the proof of Lemma 6.1. The check of the order relation between thresholds and target equilibrium values can be performed separately for every dimension $i$, while bearing in mind that $|R(D)|=$ $2^{n}$ in the worst case (compare $D^{7}$ in the example).

As an example, consider the computation of $V\left(D^{1}, \Psi\left(D^{1}\right)\right)$ in the case of the qualitative PL model of Figure 10. $D^{1}$ is a regulatory domain defined by

$$
D^{1}=\left\{\left(x_{a}, x_{b}\right)^{\prime} \in \mathbb{R}^{2} \mid 0 \leq x_{a}<\theta_{a}^{1}, 0 \leq x_{b}<\theta_{b}^{1}\right\},
$$

while $\phi\left(D^{1}\right)=\left(\kappa_{a} / \gamma_{a}, \kappa_{b} / \gamma_{b}\right)^{\prime}$ (Figure 6). From the parameter values we infer that $\kappa_{a} / \gamma_{a}>\theta_{a}^{1}$ and $\kappa_{b} / \gamma_{b}>\theta_{b}^{1}$. As a consequence, it follows with Proposition 6.1 that $V\left(D^{1}, \Psi\left(D^{1}\right)\right)=\{(1,1)\}$. The resulting qualitative state is $Q S^{1}=\left\langle D^{1},\{(1,1)\}\right\rangle$. Another example is the computation of $V\left(D^{2}, \Psi\left(D^{2}\right)\right)$, where $D^{2}$ is a switching domain, with switching variable $x_{b}$. More precisely,

$$
D^{2}=\left\{\left(x_{a}, x_{b}\right)^{\prime} \in \mathbb{R}^{2} \mid 0 \leq x_{a}<\theta_{a}^{1}, x_{b}=\theta_{b}^{1}\right\} .
$$

$R\left(D^{2}\right)=\left\{D^{1}, D^{3}\right\}$ and $\phi\left(D^{1}\right)=\left(\kappa_{a} / \gamma_{a}, \kappa_{b} / \gamma_{b}\right)^{\prime}$ and $\phi\left(D^{3}\right)=\left(0, \kappa_{b} / \gamma_{b}\right)^{\prime}$. Because $\kappa_{b} / \gamma_{b}>\theta_{b}^{1}$, and hence $\phi_{b}\left(D^{1}\right)>\theta_{b}^{1}$ and $\phi_{b}\left(D^{3}\right)>\theta_{b}^{1}$, it can be inferred from Proposition 6.2 that $V\left(D^{2}, \Psi\left(D^{2}\right)\right)=\{\}$, and hence $Q S^{2}=\left\langle D^{2},\{\}\right\rangle$. A third example concerns the computation of $V\left(D^{4}, \Psi\left(D^{4}\right)\right)$, where $D^{4}$ is a switching domain with switching variable $x_{b}$. We have

$$
D^{4}=\left\{\left(x_{a}, x_{b}\right)^{\prime} \in \mathbb{R}^{2} \mid 0 \leq x_{a}<\theta_{a}^{1}, x_{b}=\theta_{b}^{2}\right\} .
$$

Using $R\left(D^{4}\right)=\left\{D^{3}, D^{5}\right\}$, we find by means of Proposition 6.2 that $V\left(D^{4}, \Psi\left(D^{4}\right)\right) \neq$ 
\{\} . Given that $\phi\left(D^{3}\right)=\left(0, \kappa_{b} / \gamma_{b}\right)^{\prime}$ and $\phi\left(D^{5}\right)=(0,0)^{\prime}$, the parameter values imply $0<\theta_{b}^{2}<\kappa_{b} / \gamma_{b}$. Application of Proposition 6.3 yields $V\left(D^{4}, \Psi\left(D^{4}\right)\right)=\{(0,0)\}$, so that the qualitative state associated with $D^{4}$ is $Q S^{4}=\left\langle D^{4},\{(0,0)\}\right\rangle$.

\subsection{Computation of transitions between qualitative states}

Let $Q S$ and $Q S^{\prime}$ be qualitative states associated with domains $D$ and $D^{\prime}$, such that $D^{\prime} \in A(D)$. In Section 4.2, the conditions for a transition from $Q S$ to $Q S^{\prime}$ to occur were discussed, consisting of the existence of a solution trajectory starting in $D$ and remaining in this domain on some finite time-interval until reaching $D^{\prime}$. Like in Section 6.1, we will approximate $\Phi(D)$ by $\Psi(D)$. In order for this approximation to make sense, we would like to hold the crucial property of $\Phi(D)$, that every $\phi \in \Phi(D) \cap D$ is an equilibrium point of the system, to hold for $\Psi(D)$ as well. This presupposes that we also formulate a hyperrectangular approximation for $\boldsymbol{H}(\boldsymbol{x})$, thus redefining the dynamics of the system according to (14). More specifically, we set for every $\boldsymbol{x} \in D$,

$$
\boldsymbol{H}(\boldsymbol{x})=\left\{\boldsymbol{\mu}^{D}-\boldsymbol{\nu}^{D} \boldsymbol{x}\right\}, \text { if } D \text { is a regulatory domain, }
$$

and

$$
\boldsymbol{H}(\boldsymbol{x})=\overline{\operatorname{rect}}\left(\left\{\boldsymbol{\mu}^{D^{\prime}}-\boldsymbol{\nu}^{D^{\prime}} \boldsymbol{x} \mid D^{\prime} \in R(D)\right\}\right) \text {, if } D \text { is a switching domain. }
$$

It now follows that every $\psi \in \Psi(D) \cap D$ is an equilibrium point of the system. The hyperrectangular approximations of $\Phi(D)$ and $\boldsymbol{H}(\boldsymbol{x})$ underlie the following rules for computing the possible transitions from a qualitative state.

Proposition 6.4: Given domains $D$ and $D^{\prime}$, let $V\left(D, D^{\prime}\right)=\{\boldsymbol{w}\}$ be the sign vector denoting their relative position. Furthermore, let $D^{\prime} \in A(D)$. There is a transition from $Q S=\langle D, V(D, \Psi(D))\rangle$ to $Q S^{\prime}=\left\langle D^{\prime}, V\left(D^{\prime}, \Psi\left(D^{\prime}\right)\right)\right\rangle$, iff

1. $V(D, \Psi(D)) \neq\{\}$, and

2. there is some $v \in V(D, \Psi(D))$, such that $v_{i} w_{i}=1$ for every $x_{i}, 1 \leq i \leq n$, that is a switching variable in $D^{\prime}$, but not in $D$.

Proof: We first prove sufficiency of the conditions 1 and 2 to yield a transition. Let $x_{i}$ be a switching variable in $D^{\prime}$, but not in $D$, which means that $D_{i}^{\prime}=\left\{\theta_{i}^{k_{i}}\right\}$, for some $k_{i}, 1 \leq k_{i} \leq p_{i}$. Let $w_{i}=1$. The conditions imply that there is some $\boldsymbol{\psi} \in \Psi(D)$, such that, for all $d \in D_{i}, d<\theta_{i}^{k_{i}}<\psi_{i}$. For $w_{i}=-1$, we obtain $\psi_{i}<\theta_{i}^{k_{i}}<d$. For every $\boldsymbol{x}_{0} \in D$ and $\boldsymbol{\psi} \in \Psi(D)$, there exist solutions $\boldsymbol{\xi}\left(t, \boldsymbol{x}_{0}, \boldsymbol{\theta}, \boldsymbol{\kappa}, \boldsymbol{\gamma}\right)$ in $D$ monotonically converging towards $\boldsymbol{\psi}$ (Appendix A). As a consequence, the solutions will reach one or more hyperplanes $x_{i}=\theta_{i}^{k_{i}}$, where $x_{i}$ is a switching variable in $D^{\prime}$, but not in $D$. We now choose $\boldsymbol{x}_{0}$, such that the corresponding solutions $\boldsymbol{\xi}(t)$ pass through the intersection of the threshold hyperplanes $x_{i}=\theta_{i}^{k_{i}}$. This implies that the trajectories reach $D^{\prime}$ from $D$. Because (24) holds for the models that interest us, the condition at the end of Section 4.2 is satisfied for $\Psi(D)$, and at least some solutions reach $D^{\prime}$ from $D$ in finite time. This results in a transition from $Q S$ to $Q S^{\prime}$. 
Necessity is proven by contraposition, that is, by showing that, if the conditions 1 and 2 are not satisfied, then we cannot have a transition. If condition 1 is false, then a transition is certainly not possible. Suppose that condition 2 is false. That is, for some $i$, such that $x_{i}$ is a switching variable in $D^{\prime}$, but not in $D, v_{i} w_{i} \neq 1$ for all $\boldsymbol{v} \in V(D, \Psi(D))$. We have $D_{i}^{\prime}=\left\{\theta_{i}^{k_{i}}\right\}$, for some $k_{i}, 1 \leq k_{i} \leq p_{i}$. If $w_{i}=1$, then $\psi_{i}<\theta_{i}^{k_{i}}$, whereas if $w_{i}=-1$, then $\theta_{i}^{k_{i}}<\psi_{i}$, for all $\boldsymbol{\psi} \in \Psi(D)$. For all solutions $\boldsymbol{\xi}\left(t, \boldsymbol{x}_{0}, \boldsymbol{\theta}, \boldsymbol{\kappa}, \boldsymbol{\gamma}\right)$ in $D$, it holds that $\xi_{i}(t)$ monotonically converges towards the projection of $\Psi(D)$ on $\Omega_{i}$ (Appendix A). As a consequence, no solution can reach the threshold hyperplane $x_{i}=\theta_{i}^{k_{i}}$, and hence there does not exist a transition from $Q S$ to $Q S^{\prime}$.

Proposition 6.5: Given domains $D$ and $D^{\prime}$, let $V\left(D, D^{\prime}\right)=\{\boldsymbol{w}\}$ be the sign vector denoting their relative position. Furthermore, let $D \in A\left(D^{\prime}\right)$. There is a transition from $Q S=\langle D, V(D, \Psi(D))\rangle$ to $Q S^{\prime}=\left\langle D^{\prime}, V\left(D^{\prime}, \Psi\left(D^{\prime}\right)\right)\right\rangle$, iff

1. $V\left(D^{\prime}, \Psi\left(D^{\prime}\right)\right) \neq\{\}$, and

2. there is some $\boldsymbol{v} \in V\left(D^{\prime}, \Psi\left(D^{\prime}\right)\right)$, such that $v_{i} w_{i} \neq-1$ for every $x_{i}, 1 \leq i \leq n$, that is a switching variable in $D$, but not in $D^{\prime}$.

The proof is similar to that of Proposition 6.4 and will be omitted.

Using qualitative states $\langle D, V(D, \Psi(D))\rangle$ instead of $\langle D, V(D, \Phi(D))\rangle$ has the consequence that, for all combinations of parameter values consistent with the inequality constraints in the qualitative PL model, the set of transitions from a qualitative state to other qualitative states is unique. Again, this is not guaranteed for qualitative states $\langle D, V(D, \Phi(D))\rangle$.

LEMMA 6.2: For all quantitative PL models subsumed by a qualitative PL model, every qualitative state $Q S=\langle D, V(D, \Psi(D))\rangle$ has a unique set of outgoing transitions $\left\{\left\langle Q S, Q S^{\prime}\right\rangle \mid Q S^{\prime} \in \mathcal{Q S}\right\}$.

Proof: Lemma 6.1 states that under the conditions of the proposition, every domain $D$ is associated with a unique qualitative state $Q S=\langle D, V(D, \Psi(D))\rangle$. As a consequence of Propositions 6.4 and 6.5, the set of transitions from $Q S$ is unique.

Given a qualitative state $Q S=\langle D, V(D, \Psi(D))\rangle$ obtained from a qualitative PL model, Propositions 6.4 and 6.5 allow the transitions from this state to be computed symbolically, by checking the two conditions in the rule that applies. In the worst case, a domain is in the boundary of $3^{n}-1$ other domains (compare $D^{7}$ in the example). The maximum difference in the number of switching variables between two domains is $n$ (compare $D^{1}$ and $D^{7}$ in the example). The computation of transitions from a qualitative state is therefore exponential in the number of variables.

We will illustrate the transition rules by means of the two examples in Figure 11, again derived from the qualitative PL model in Figure 10. Consider the possible transitions from the qualitative state $Q S^{3}$ associated with regulatory domain $D^{3}$ to qualitative states associated with the boundary domains $A\left(D^{3}\right)=\left\{D^{2}, D^{4}, D^{7}, D^{8}, D^{9}\right\}$ 
in (a). We have to verify whether the conditions 1 and 2 of Proposition 6.4 are verified. $V\left(D^{3}, \Psi\left(D^{3}\right)\right)$ is calculated to be $\{(0,1)\}$, while $V\left(D^{3}, D^{4}\right)$ equals $\{(0,1)\}$. With $x_{b}$ a switching variable in $D^{4}$, but not in $D^{3}$, we find that conditions 1 and 2 are satisfied. Consequently, a transition from $Q S^{3}$ to $Q S^{4}$ is generated. Transitions from $Q S^{3}$ to the other candidate successor states are ruled out, because they violate condition 2 . Because $D^{3}$ is not in the boundary of any domain, Proposition 6.5 cannot be applied.

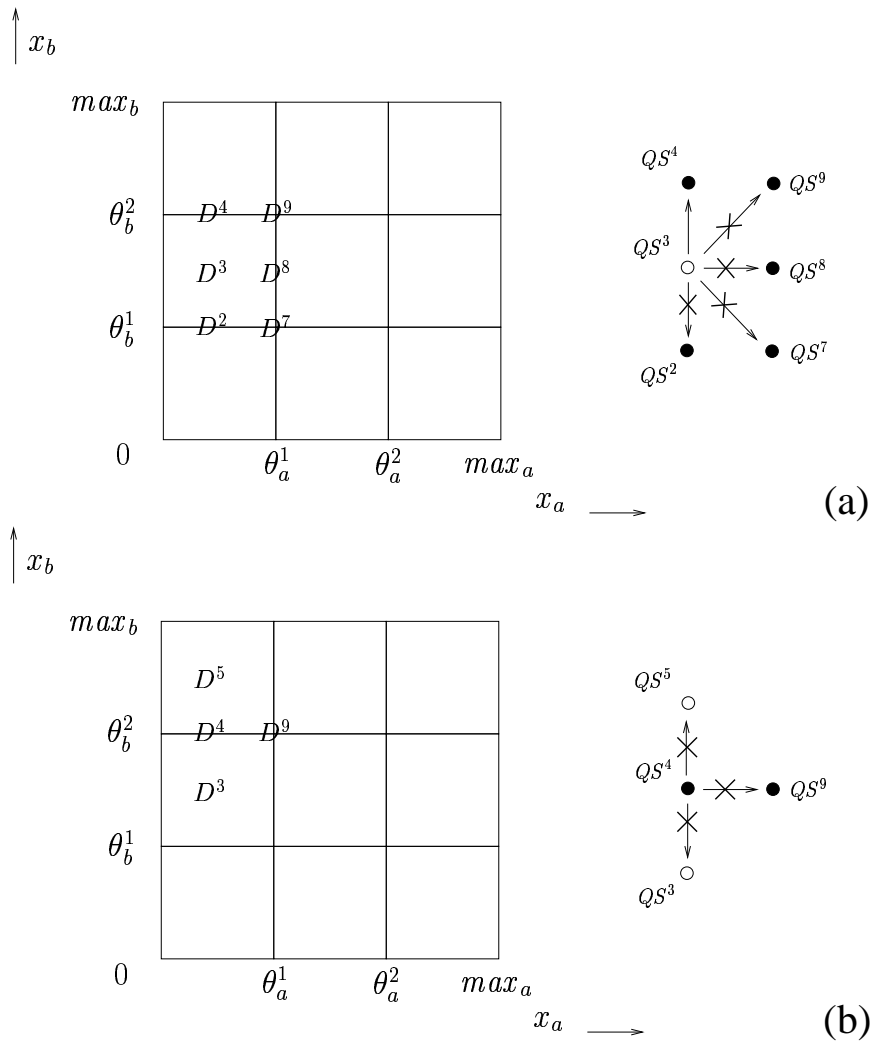

Figure 11: Possible and impossible ( $\times$ ) transitions from the qualitative states (a) $Q S^{3}$ and (b) $Q S^{4}$, as determined by the transition rules in Propositions 6.4 and 6.5 .

In Figure 11(b), the transitions from the qualitative state $Q S^{4}=\left\langle D^{4},\{(0,0)\}\right\rangle$ to the states associated with the boundary domain $A\left(D^{4}\right)=\left\{D^{9}\right\}$ are investigated. The transition to $Q S^{9}=\left\langle D^{9},\{\}\right\rangle$ is excluded, because condition 2 of Proposition 6.4 is not satisfied. In addition, we consider transitions from $Q S^{4}$ to qualitative states associated with domains that have $D^{4}$ in their boundary. Proposition 6.5 is valid for these cases. As can be verified in the figure, $D^{4} \in A\left(D^{3}\right)$ and $D^{4} \in A\left(D^{5}\right) . D^{3}$ and $D^{5}$ are regulatory domains, so condition 1 is trivially satisfied. However, with $x_{b}$ being a switching variable in $D^{4}$, but not in $D^{3}$ and $D^{5}$, condition 2 is satisfied in neither case. We therefore conclude that there are no transitions from $Q S^{4}$. 


\subsection{Computation of state transition graph}

Given a qualitative PL model, we can generate the qualitative states and transitions between qualitative states by means of the Propositions 6.1 to 6.5. This results in a state transition graph with the following important property.

THEOREM 6.1: Under the approximation of $\Phi(D)$ by $\Psi(D)$, all quantitative PL models subsumed by a qualitative PL model have the same state transition graph.

The theorem, a direct consequence of Lemmas 6.1 and 6.2, implies that, under the approximation of $\Phi(D)$ by $\Psi(D)$, the entire set of quantitative PL models subsumed by the qualitative PL model can be analyzed in one stroke. The state transition graph generated from the qualitative PL model summarizes the qualitative dynamics of every quantitative PL model having parameter values consistent with the inequality constraints. In the case of the example network, using the model in Figure 10, the state transition graph in Figure 8 is obtained.

The number of qualitative states in a state transition graph exponentially grows with the dimension $n$ of the system. As a consequence, the graph may become forbiddingly large for genetic regulatory networks with more than a few genes. For many purposes, it is not necessary to generate the complete state transition graph though. In fact, it often is sufficient to know which qualitative states are reachable from a given initial qualitative state, that is, which qualitative behaviors the system can exhibit when starting from the initial qualitative state. The generation of the reachable part of the state transition graph will be called qualitative simulation, by analogy with [39]. If no misunderstanding is possible, we often refer to this reachable part of the state transition graph as the state transition graph.

The qualitative simulation algorithm can be summarized as follows. Given an initial domain $D^{0}$, the simulation algorithm computes the initial qualitative state $Q S^{0}$ from the qualitative PL model, and then determines all possible transitions from $Q S^{0}$ to successor qualitative states by means of the rules of the previous subsection. The generation of successor states is repeated in a recursive manner until all qualitative states reachable from the initial qualitative state have been found. A formal description of the simulation algorithm can be found in [11]. An example of a state transition graph obtained through qualitative simulation is given in Figure 12, showing the qualitative states reachable from the qualitative state $Q S^{1}$.

The simulation method has been implemented in Java 1.3, in a program called GNA (Genetic Network Analyzer) [10]." The program reads and parses input files specifying the model of the system (state equations, threshold and equilibrium inequalities) and the initial domain. From this information it produces a state transition graph. GNA is accessible through a graphical user-interface, which allows the network of interactions between genes to be displayed, as well as the state transition graph resulting from the simulation. In addition, the user can analyze the qualitative equilibrium

$\|_{\text {GNA }}$ is available for non-profit academic research purposes at http://wwwhelix.inrialpes.fr/gna. 

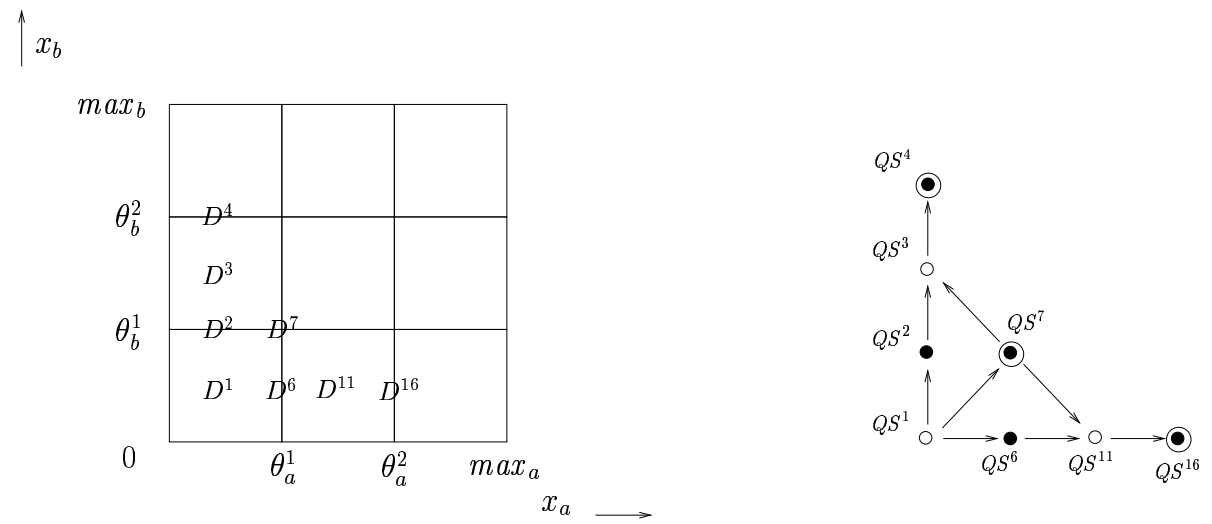

Figure 12: Phase space and state transition graph obtained by qualitative simulation using the model in Figure 10. The state transition graph contains the qualitative states reachable from the initial qualitative state $Q S^{1}$.

states and qualitative cycles with their attraction sets, and focus on selected qualitative behaviors in order to study the temporal evolution of protein concentrations in more detail.

\section{Properties of qualitative simulation}

Given a qualitative PL model and an initial regulatory domain $D^{0}$, qualitative simulation generates a state transition graph which, under the approximation of $\Phi(D)$ by $\Psi(D)$, describes the qualitative dynamics of the system for all quantitative PL models subsumed by the qualitative PL model. In this section, we investigate the consequences of the use of this approximation, by comparing the state transition graph obtained through qualitative simulation, using $\Psi(D)$ instead of $\Phi(D)$, with the state transition graphs that would be obtained using $\Phi(D)$.

\subsection{Qualitative simulation is sound}

In order to clarify the discussion below, we will explicitly distinguish between two sets of state transition graphs. On the one hand, we will be interested in $\mathcal{G}_{\Phi}$, the set of state transition graphs obtained for quantitative PL models subsumed by the qualitative PL model, using $\Phi(D)$. On the other hand, we consider the set of state transition graphs $\mathcal{G}_{\Psi}$ obtained by means of the approximation $\Psi(D)$ of $\Phi(D)$. From Theorem 6.1 it follows that this set has a single element. More particularly, we will focus on $\mathcal{G}_{\Phi}^{0}$ and $\mathcal{G}_{\Psi}^{0}$, which contain the state transition graphs comprising all qualitative states reachable from initial qualitative states $Q S_{\Phi}^{0}=\left\langle D^{0}, V\left(D^{0}, \Phi\left(D^{0}\right)\right)\right\rangle$ and $Q S_{\Psi}^{0}=$ $\left\langle D^{0}, V\left(D^{0}, \Psi\left(D^{0}\right)\right)\right\rangle$, respectively. The following theorem allows us to evaluate the consequences of using $\Psi(D)$ rather than $\Phi(D)$.

THEOREM 7.1: Let $G_{\Psi}^{0}=\left\langle\mathcal{Q S}_{\Psi}, \mathcal{T}_{\Psi}\right\rangle \in \mathcal{G}_{\Psi}^{0}$ be the state transition graph obtained through qualitative simulation. Every $G_{\Phi}^{0}=\left\langle\mathcal{Q S}_{\Phi}, \mathcal{T}_{\Phi}\right\rangle \in \mathcal{G}_{\Phi}^{0}$ is contained in $G_{\Psi}^{0}$, that is, 
1. If $Q S_{\Phi}=\langle D, V(D, \Phi(D))\rangle \in \mathcal{Q} \mathcal{S}_{\Phi}$, then $Q S_{\Psi}=\langle D, V(D, \Psi(D))\rangle \in \mathcal{Q} \mathcal{S}_{\Psi}$, and

2. If $T_{\Phi}=\left\langle Q S_{\Phi}, Q S_{\Phi}^{\prime}\right\rangle \in \mathcal{T}_{\Phi}$, then $T_{\Psi}=\left\langle Q S_{\Psi}, Q S_{\Psi}^{\prime}\right\rangle \in \mathcal{T}_{\Psi}$.

Proof: The theorem is a consequence of the redefinition of $\boldsymbol{H}(\boldsymbol{x})$, presupposed by the approximation of $\Phi(D)$ by $\Psi(D)$ (Section 6.2). Every $Q S_{\Phi} \in \mathcal{Q} \mathcal{S}_{\Phi}$ is reachable from $Q S_{\Phi}^{0}$ through one or more transitions, by definition of $G_{\Phi}^{0}$. By comparing (16) with (31), we see that the set of solutions of the approximate system includes the set of solutions of the original system. It directly follows that there is also a qualitative behavior leading from $Q S_{\Psi}^{0}$ to $Q S_{\Psi}$, and hence $Q S_{\Psi} \in \mathcal{Q} \mathcal{S}_{\Psi}$. If $T_{\Phi} \in \mathcal{T}_{\Phi}$, then $T_{\Psi} \in \mathcal{T}_{\Psi}$, as a consequence of 1 and the observation that the set of solutions of the approximate system includes the set of solutions of the original system.

The theorem expresses that the approximation of $\Phi(D)$ by $\Psi(D)$ is conservative. Formulated in a different way, it means that qualitative simulation does not miss any of the qualitative behaviors permitted by some quantitative PL model subsumed by the qualitative PL model. This means that the propositions in Section 4.3 can be used to relate the state transition graph obtained through qualitative simulation to the solutions of quantitative PL models. In particular, Proposition 4.3 and Theorem 7.1 imply that for all parameter values satisfying the inequality constraints in the qualitative PL model, the set of solutions passing through a finite sequence of domains on a finite time-interval is covered by the state transition graph. By analogy with [39], the qualitative simulation method is said to be sound.

\subsection{Qualitative simulation is incomplete}

The converse of Theorem 7.1 is not true: there may be some $G_{\Phi}^{0} \in \mathcal{G}_{\Phi}^{0}$, such that $G_{\Psi}^{0} \in$ $\mathcal{G}_{\Psi}^{0}$ is not contained in $G_{\Phi}^{0}$. This will be illustrated by means of a counterexample.

The network in Figure 13 consists of two genes, $a$ and $b$, which encode the proteins $\mathrm{A}$ and $\mathrm{B}$, respectively. The proteins form a heterodimer $\mathrm{A} \cdot \mathrm{B}$ repressing the expression of both genes. The qualitative PL model is shown in Figure 14. It is assumed that the heterodimer represses the two genes at the same threshold concentration. The phase space associated with the model consists of four regulatory domains and five switching domains (Figure 15(a)). When simulating the network from the initial qualitative state associated with the regulatory domain $D^{1}$, in which both $x_{a}$ and $x_{b}$ are below their threshold concentration, we obtain the state transition graph shown in Figure 15(b).

The results that would be obtained if $\Phi(D)$ instead of its approximation $\Psi(D)$ were used are shown in Figure 16. In this case, the quantitative PL models subsumed by the qualitative PL model are associated with one of three different state transition graphs, depending on the precise parameter values chosen. As can be directly verified, the state transition graph obtained through qualitative simulation contains all three state transition graphs in Figure 16, as was to be expected on the basis of Theorem 7.1. However, it is contained in none of these graphs. In this example, the approximation 


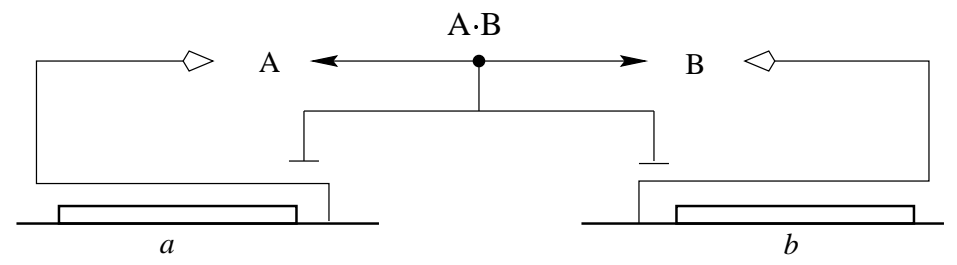

Figure 13: Example of a genetic regulatory network of two genes ( $a$ and $b$ ) coding for a regulatory protein (A and B). The regulatory proteins form a heterodimer A.B.

State equation for gene $a$ : $\dot{x}_{a}=\kappa_{a}\left(1-s^{+}\left(x_{a}, \theta_{a}^{1}\right) s^{+}\left(x_{b}, \theta_{b}^{1}\right)\right)-\gamma_{a} x_{a}$

Threshold inequalities: $0<\theta_{a}^{1}<\max _{a}$

Equilibrium inequalities: $\theta_{a}^{1}<\frac{\kappa_{a}}{\gamma_{a}}<\max _{a}$

State equation for gene $b: \dot{x}_{b}=\kappa_{b}\left(1-s^{+}\left(x_{a}, \theta_{a}^{1}\right) s^{+}\left(x_{b}, \theta_{b}^{1}\right)\right)-\gamma_{b} x_{b}$

Threshold inequalities: $0<\theta_{b}^{1}<\max _{b}$

Equilibrium inequalities: $\theta_{b}^{1}<\frac{\kappa_{b}}{\gamma_{b}}<\max _{b}$

Figure 14: State equations, threshold inequalities, and equilibrium inequalities for the proteins in the network of Figure 13.
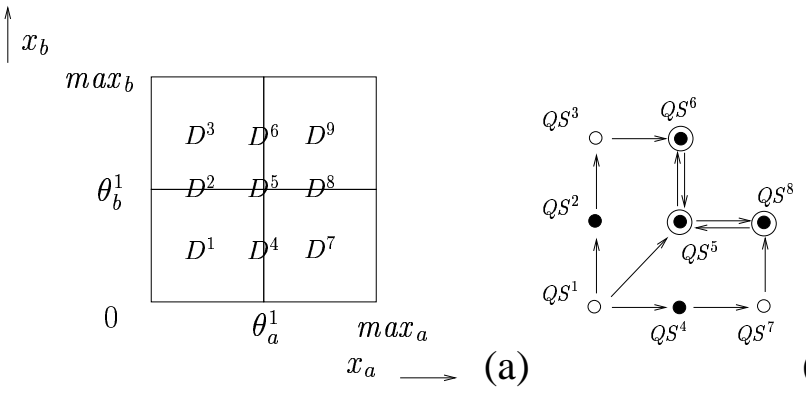

(a)

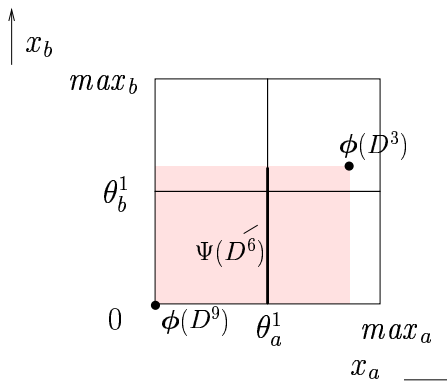

(c)

Figure 15: (a) Phase space box for the genetic regulatory network described by the qualitative PL model in Figure 14. (b) State transition graph resulting from a qualitative simulation of the system starting in $D^{1}$. (c) Determination of the relative position of $D^{6}$ and $\Psi\left(D^{6}\right)$. The shaded area represents $\overline{r e c t}\left(\left\{\phi\left(D^{3}\right), \phi\left(D^{9}\right)\right\}\right)$.

leads to the faulty prediction that the system has three qualitative equilibrium states, 
whereas in fact it has only one, whose identity varies with the parameter values chosen.

In order to explain this result, consider the computation of the qualitative state associated with the switching domain $D^{6} . R\left(D^{6}\right)=\left\{D^{3}, D^{9}\right\}$, while $\phi\left(D^{3}\right)=$ $\left(\kappa_{a} / \gamma_{a}, \kappa_{b} / \gamma_{b}\right)^{\prime}$ and $\phi\left(D^{9}\right)=(0,0)^{\prime}$. Using the equilibrium inequalities in Figure 14, we find $V\left(D^{6}, \Psi\left(D^{6}\right)\right)=\{(0,-1),(0,0)\}$, where $\Psi\left(D^{6}\right)$ is the intersection of the smallest rectangle including $\phi\left(D^{3}\right)$ and $\phi\left(D^{9}\right)$, and the threshold boundary $x_{a}=\theta_{a}^{1}$ (Figure 15(c)). $Q S^{6}$ is predicted to be a qualitative equilibrium state, because $(0,0) \in$ $V\left(D^{6}, \Psi\left(D^{6}\right)\right)$. However, $(0,-1) \in V\left(D^{6}, \Psi\left(D^{6}\right)\right)$ allows a transition to $Q S^{5}$, associated with $D^{5} \in A\left(D^{6}\right)$, to occur as well.

Figure 16 shows that $\Psi\left(D^{6}\right)$ is actually an overapproximation of $\Phi\left(D^{6}\right)$. The smallest closed convex set $\overline{c o}\left(\left\{\phi\left(D^{3}\right), \phi\left(D^{9}\right)\right\}\right)$ is not $\overline{r e c t}\left(\left\{\phi\left(D^{3}\right), \phi\left(D^{9}\right)\right\}\right)$, but rather the linear segment connecting the two target equilibria. As a consequence, $\Phi\left(D^{6}\right)$ is a single point in $\Omega$, located at the intersection of the linear segment connecting $\phi\left(D^{3}\right)$ and $\phi\left(D^{9}\right)$, and the threshold boundary $x_{a}=\theta_{a}^{1}$. Depending on the exact position of $\phi\left(D^{3}\right)$ in $D^{9}$, the linear segment crosses (a) $D^{6}$, (b) $D^{5}$, or (c) $D^{4}$. Each configuration in Figure 16(a)-(c) implies an additional constraint on the parameter values. In particular, we have
(a) $\frac{\gamma_{a}}{\kappa_{a}} \frac{\kappa_{b}}{\gamma_{b}}>\frac{\theta_{b}^{1}}{\theta_{a}^{1}}$
(b) $\frac{\gamma_{a}}{\kappa_{a}} \frac{\kappa_{b}}{\gamma_{b}}=\frac{\theta_{b}^{1}}{\theta_{a}^{1}}$
and
(c) $\frac{\gamma_{a}}{\kappa_{a}} \frac{\kappa_{b}}{\gamma_{b}}<\frac{\theta_{b}^{1}}{\theta_{a}^{1}}$.

For (a) we have $V\left(D^{6}, \Phi\left(D^{6}\right)\right)=\{(0,0)\}$, for (b) $V\left(D^{6}, \Phi\left(D^{6}\right)\right)=\{(0,-1)\}$, and for (c) $V\left(D^{6}, \Phi\left(D^{6}\right)\right)=\{(0,-1)\}$. In (b) and (c) $Q S^{6}$ is not a qualitative equilibrium state, while in (a) and (b) there is no transition to $Q S^{5}$. (In (b), trajectories starting in $D^{6}$ reach $D^{5}$ only in infinite time, so there is no transition from $Q S^{6}$ to $Q S^{5}$.) For each of the three cases, we obtain a different state transition graph, as shown in Figure 16.

The example illustrates that the approximation of $\Phi(D)$ by $\Psi(D)$ may be overly conservative, in the sense that the resulting state transition graph contains qualitative behaviors that are not permitted by any of the quantitative PL models subsumed by the qualitative PL model. Some of the qualitative behaviors in Figure 15(b) do not occur in any of the state transition graphs in Figure 16(a)-(c), such as $\left(Q S^{1}, Q S^{4}, Q S^{7}, Q S^{8}\right.$, $\left.Q S^{5}, Q S^{8}\right)$. This implies that an absolutely continuous function passing through the corresponding sequence of domains on a finite time-interval is not a solution for any parameter values satisfying the inequality constraints in the qualitative PL model (Proposition 4.3). Qualitative simulation is incomplete, in the terminology of [39].

\section{Discussion}

We have presented a method for the qualitative simulation of genetic regulatory networks described by a class of piecewise-linear (PL) differential equations that has been well-studied in mathematical biology. The method allows the behavior emerging from large and complex networks of genetic regulatory interactions to be predicted in a qualitative manner. In the accompanying paper, we describe a model of the network 


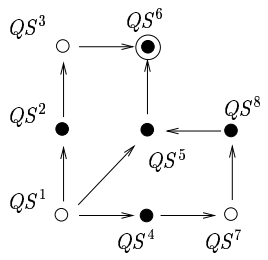

(a)

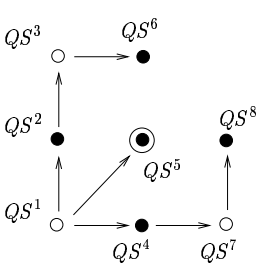

(b)

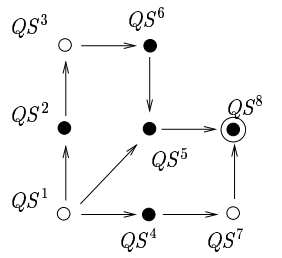

(c)

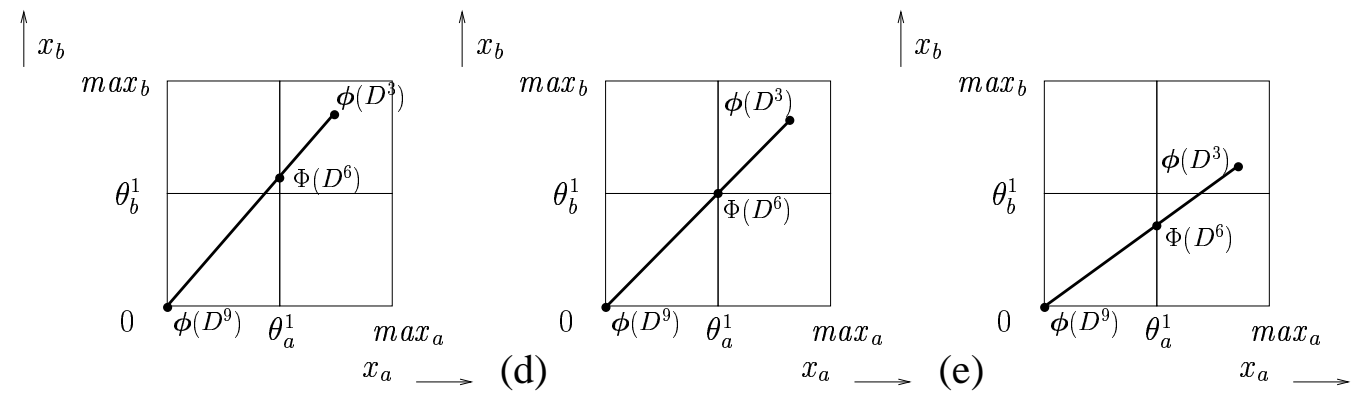

Figure 16: (a)-(c) State transition graphs obtained for the qualitative PL model in Figure 14, containing qualitative states reachable from $Q S^{1} . \Phi(D)$ instead of its approximation $\Psi(D)$ has been used. The state transition graphs correspond to the following additional constraints on the parameter values: (a) $\left(\kappa_{b} / \gamma_{b}\right) /\left(\kappa_{a} / \gamma_{a}\right)>\theta_{b}^{1} / \theta_{a}^{1}$, (b) $\left(\kappa_{b} / \gamma_{b}\right) /\left(\kappa_{a} / \gamma_{a}\right)=\theta_{b}^{1} / \theta_{a}^{1}$, and (c) $\left(\kappa_{b} / \gamma_{b}\right) /\left(\kappa_{a} / \gamma_{a}\right)<\theta_{b}^{1} / \theta_{a}^{1}$. (d)(f) Different constraints lead to different relative positions of $D^{6}$ and $\Phi\left(D^{6}\right)$, and hence to different qualitative states $Q S^{6}$.

underlying the initiation of sporulation in B. subtilis, and we compare predictions obtained through simulation with observations of the behavior of wild type and mutant bacteria [9]. The application of the qualitative simulation method is supported by a computer tool, called Genetic Network Analyzer (GNA) [10].

The PL models employed in this paper are based on step function approximations of the regulatory interactions involved in the synthesis and degradation of proteins. The step functions provide a succinct description of the regulatory logic, while abstracting from the details of molecular interactions. The biological validity of the step function expressions derives from experimental evidence that the activation of a gene, as a function of the concentration of a regulatory protein, often follows a steep sigmoidal curve $[56,71]$. That is, below a certain threshold concentration of the protein, the gene will be hardly expressed at all, whereas above this threshold its expression rapidly saturates. Recent experimental studies have shown that some aspects of the qualitative dynamics of genetic regulatory networks synthesized in vivo correspond well with the predictions obtained from mathematical models based on switch-like approximations of regulatory interactions $[5,17,21]$.

The use of step functions gives rise to discontinuities in the right-hand side of the differential equations, which may lead to nontrivial mathematical problems, as illustrated in Figure 4. Several ways to deal with the step function discontinuities have been proposed in the literature. The application of the PL models can be restricted to systems without autoregulation, which excludes situations of the type described 
in Figure 4(b) [25]. Alternatively, when a trajectory arrives at a switching domain from which it cannot be continued, it may simply be stipulated to come to a dead stop [52]. Another solution, based on an idea of Plahte et al. [47, 52], consists of avoiding the discontinuities altogether, by replacing the step functions $s^{+}\left(x_{j}, \theta_{j}\right)$ by so-called logoid functions $l^{+}\left(x_{j}, \theta_{j}, \delta\right)$ that monotonically increase from 0 to 1 in a $\delta$-interval around the threshold $\theta_{j}$. The logoid functions approach their step function homologues as $\delta \rightarrow 0$.

Each of the above solutions is unsatisfactory in some way. In the first place, autoregulation is an ubiquitous feature in genetic regulatory networks [64]. ${ }^{* *}$ Ignoring trajectories that cannot be continued in a switching domain will cause important behavioral properties of regulatory systems to be missed, like the equilibrium points located in threshold boundaries in the example. The use of logoid functions instead of step functions is attractive at first sight, but leads to nonlinear differential equation models that are difficult to treat in a qualitative way. Here, we have adopted another solution, based on an approach to deal with differential equations with discontinuous right-hand sides originally proposed by Filippov [19]. This approach, recently applied to PL models of the form (2), has the advantage of putting no restrictions on the class of genetic regulatory networks that can be handled, while explicitly defining the behavior of the system in the threshold hyperplanes by means of simple-to-analyze PL models [31].

The qualitative dynamics of genetic regulatory networks described by the PL models (2) can be summarized by means of a state transition graph. Each qualitative state in the graph corresponds to a domain in the phase space where the system behaves in a qualitatively distinct manner, while a transition between two qualitative states corresponds to solution trajectories that start in one domain and reach the other, without passing through an intermediate domain. Qualitative equilibrium states and qualitative cycles in a state transition graph point at equilibrium points and limit cycles of the system, while a qualitative behavior represents the qualitative shape of solutions. The qualitative nature of the state transition graph is well-adapted to measurement techniques in genomics, which currently have limited quantitative precision, but are able to detect qualitative changes in gene expression over time.

Instead of precise numerical values, we use inequality constraints on the values of threshold and rate parameters. The resulting qualitative PL model corresponds to a set of quantitative PL models. Whereas precise numerical values for the parameters are usually not available, the choice of appropriate threshold and equilibrium inequalities can be based on biological data, or is at least strongly constrained by the latter. If the choice of inequality constraints is not unambiguously determined by the data, the consequences of opting for one combination of constraints rather than another can be explored by simulating the system for each of the alternatives.

The aim of qualitative simulation is to generate the possible state transition graphs for the quantitative PL models subsumed by a qualitative PL model. The state transi-

\footnotetext{
${ }^{* *}$ In fact, the network shown in Figure 1 is a simplified version of the molecular switch determining
} the response of $E$. coli to phage $\lambda$ infection [56]. 
tion graphs contain all qualitative states reachable from a given initial state through one or more transitions. We have used the hyperrectangular approximation $\Psi(D)$ of $\Phi(D)$, which guarantees that in the region of the parameter space defined by the inequalities in the qualitative PL model, all quantitative PL models yield the same state transition graph. This graph can be obtained through symbolic computation instead of numerical simulation. Qualitative simulation has been shown to be sound, in that to every solution of a quantitative PL model subsumed by the qualitative PL model corresponds a qualitative behavior in the state transition graph. The soundness of qualitative simulation may help in checking the robustness of simulation results to changes in parameter values $[2,3,4,49,69]$. If a certain behavior is not covered by the state transition graph, one can be sure that it will not occur for any of the parameter values consistent with the threshold and equilibrium inequalities. Although the simulation algorithm is sound, it is not complete. This implies that the transition graph may contain qualitative behaviors that do not correspond to any solution of a quantitative PL model satisfying the inequality constraints in the qualitative PL model.

The lack of quantitative information on kinetic parameters and molecular concentrations has stimulated an interest in methods for modeling and simulation developed in the field of qualitative reasoning (QR), most notably QSIM [39] and QPT [20]. Methods similar in spirit have been proposed in the hybrid systems community $[22,23]$. QR methods have been applied to the regulation of tryptophan synthesis [33] and $\lambda$ phage growth [32] in E. coli, and to the regulation of the transcription factor families AP-1 and NF- $\kappa$ B in different classes of animals [68]. A major problem with existing QR methods is their lack of upscalability, which causes the applicability of the methods to be limited to small regulatory systems of modest complexity. As its application to the sporulation example in the accompanying paper shows, the qualitative simulation method presented here is able to deal with large and complex networks. Upscaling of the method is achieved by the use of PL models that strongly constrain the local dynamics of the system. Moreover, the representation of qualitative states and the transition rules are tailored to this class of models, in order to maximally exploit their favorable mathematical properties.

Qualitative methods for the analysis of genetic regulatory systems have been developed in mathematical biology as well, the best-known example being Boolean networks [34, 62]. Simulation of Boolean networks rests on the assumption that a gene is either active or inactive, and that genes change their activation state synchronously. For the purpose of modeling actual genetic regulatory networks, these assumptions are usually too strong. Thomas and colleagues $[66,67]$ have proposed a generalized logical method that permits multivalued activation states and asynchronic transitions. On the formal level, the method of Thomas and colleagues is related to the approach presented in this paper. In fact, Snoussi has demonstrated that the logical equations can be interpreted as an abstraction of a special case of (1), where in the production term $f_{i}(\boldsymbol{x})=\sum_{l \in L} \kappa_{i l} b_{i l}(\boldsymbol{x})$ it holds that either $b_{i l}(\boldsymbol{x})=s^{+}\left(x_{j}, \theta_{j}\right)$ or $b_{i l}(\boldsymbol{x})=s^{-}\left(x_{j}, \theta_{j}\right)$, while in the degradation term $g_{i}(\boldsymbol{x})=\sum_{l \in L} \gamma_{i l} b_{i l}(\boldsymbol{x})$ it holds that $b_{i l}(\boldsymbol{x})=1$. In addition, no two genes are regulated at the same threshold [60].

Although some ideas of the generalized logical method have been retained in the 
method presented here, in particular the inequality constraints of Section 5, which are related to the logical parameters in $[66,67]$, we have opted for differential equation models. We believe that the latter formalism is intuitively clear and of large generality. In particular, it allows for a transparent description of the behavior of the system in the threshold hyperplanes. Although for the class of PL models covered by the generalized logical method, certain patterns of logical states can be interpreted as indicating singular steady states of the system [61], a general description of the behavior of the system in the threshold hyperplanes is currently missing. The differential equation formalism has the additional advantage of facilitating the integration of quantitative data becoming available through improvements of current measurement techniques.

Qualitative simulation results in predictions of the possible qualitative behaviors of a genetic regulatory network. The interest of these predictions is that they can be directly compared with gene expression profiles obtained by means of quantitative RT-PCR or DNA microarrays. The use of predicted qualitative behaviors in combination with observed gene expression profiles allows hypothesized models of regulatory networks to be rapidly tested, even when only imprecise data is available. Along these lines, we are currently working on extensions of the method to validate and identify models of genetic regulatory networks using gene expression data. Incorporation of these extensions in the computer tool mentioned in Section 6.3 would allow the simulation method to evolve into a more general approach towards the computer-supported analysis of genetic regulatory networks.

Acknowledgments The authors would like to thank Grégory Batt, Vlastimil Krivan, Erik Plahte, Alessandro Usseglio Viretta, and an anonymous referee for their helpful comments on earlier versions of this paper. The authors acknowledge financial support from the Programme Bioinformatique inter-EPST (Ministère de la Recherche, France) and the Actions de Recherche Coopératives of INRIA.

\section{A. Monotonicity properties of piecewise-linear models}

This appendix describes monotonicity properties of PL models of the form (2).

For regulatory domains $D$, all solutions $\boldsymbol{\xi}\left(t, \boldsymbol{x}_{0}, \boldsymbol{\theta}, \boldsymbol{\kappa}, \boldsymbol{\gamma}\right)$ in $D$ monotonically converge towards $\Phi(D)$. This is not generally true for switching domains, although a weaker monotonicity property can be shown to hold. Let $\Phi(D) \neq\{\}$. Furthermore, let $\boldsymbol{\xi}\left(t, \boldsymbol{x}_{0}, \boldsymbol{\theta}, \boldsymbol{\kappa}, \boldsymbol{\gamma}\right)$ be a solution in $D$, and consider some $i, 1 \leq i \leq n$. It holds that $\xi_{i}(t)$ monotonically converges towards the projection of $\Phi(D)$ on $\Omega_{i}$,

$$
\pi_{i}(\Phi(D))=\left\{\phi_{i} \in \Omega_{i} \mid \phi \in \Phi(D)\right\},
$$

where $\xi_{i}(t)$ is assumed to start outside the projection $\left(\right.$ i.e., $\xi_{i}(0) \notin \pi_{i}(\Phi(D))$ ).

Let $\xi_{i}(t)<\min \pi_{i}(\Phi(D)), t \geq 0$. From the definition of $\Phi(D)$ in (18), it follows that $\xi_{i}(t)<\phi_{i}\left(D^{\prime}\right)=\mu_{i}^{D^{\prime}} / \nu_{i}^{D^{\prime}}$, for all $D^{\prime} \in R(D)$. By $(16), \boldsymbol{H}(\boldsymbol{\xi}(t))=$ $\left\{\sum_{D^{\prime} \in R(D)} \alpha_{D^{\prime}}\left(\boldsymbol{\mu}^{D^{\prime}}-\boldsymbol{\nu}^{D^{\prime}} \boldsymbol{\xi}(t)\right)\right\}$, with $\sum_{D^{\prime} \in R(D)} \alpha_{D^{\prime}}=1$ and $\alpha_{D^{\prime}} \geq 0$. Because

$\dot{\boldsymbol{\xi}}(t) \in \boldsymbol{H}(\boldsymbol{\xi}(t)), \dot{\xi}_{i}(t)>0$. As a consequence, $\xi_{i}(t)$ monotonically converges to- 
wards $\pi_{i}(\Phi(D))$ while being in $D$. A similar argument can be given for the case $\xi_{i}(t)>\max \pi_{i}(\Phi(D))$.

Although not all solutions in $D$ monotonically converge towards $\Phi(D)$, some can be shown to do so. Let $\Phi(D) \neq\{\}$. For every $\boldsymbol{x}_{0} \in D$ and $\boldsymbol{\phi} \in \Phi(D)$, there exist solutions $\boldsymbol{\xi}\left(t, \boldsymbol{x}_{0}, \boldsymbol{\theta}, \boldsymbol{\kappa}, \boldsymbol{\gamma}\right)$ in $D$ monotonically converging towards $\boldsymbol{\phi}$.

By (18), $\phi=\sum_{D^{\prime} \in R(D)} \alpha_{D^{\prime}} \phi\left(D^{\prime}\right)$, with every $\alpha_{D^{\prime}} \geq 0$ and $\sum_{D^{\prime} \in R(D)} \alpha_{D^{\prime}}=1$. Consider the absolutely continuous function $\boldsymbol{\xi}(t)$ that, while being in $D$, satisfies

$$
\begin{aligned}
\dot{\boldsymbol{\xi}}(t) & =\sum_{D^{\prime} \in R(D)} \frac{\alpha_{D^{\prime}}\left(\boldsymbol{\nu}^{D^{\prime}}\right)^{-1}}{\sum_{D^{\prime \prime} \in R(D)} \alpha_{D^{\prime \prime}}\left(\boldsymbol{\nu}^{D^{\prime \prime}}\right)^{-1}}\left(\boldsymbol{\mu}^{D^{\prime}}-\boldsymbol{\nu}^{D^{\prime}} \boldsymbol{\xi}(t)\right) \\
& =\sum_{D^{\prime} \in R(D)} \frac{\alpha_{D^{\prime}}}{\sum_{D^{\prime \prime} \in R(D)} \alpha_{D^{\prime \prime}}\left(\boldsymbol{\nu}^{D^{\prime \prime}}\right)^{-1}} \boldsymbol{\phi}\left(D^{\prime}\right)-\frac{1}{\sum_{D^{\prime \prime} \in R(D)} \alpha_{D^{\prime \prime}}\left(\boldsymbol{\nu}^{D^{\prime \prime}}\right)^{-1}} \boldsymbol{\xi}(t) .
\end{aligned}
$$

From (32) it follows that $\boldsymbol{\xi}(t)$ is a solution, because $\dot{\boldsymbol{\xi}}(t) \in \boldsymbol{H}(\boldsymbol{\xi}(t))$ according to (14) (the coefficients $\alpha_{D^{\prime}}\left(\boldsymbol{\nu}^{D^{\prime}}\right)^{-1} / \sum_{D^{\prime \prime} \in R(D)} \alpha_{D^{\prime \prime}}\left(\boldsymbol{\nu}^{D^{\prime \prime}}\right)^{-1}$ are non-negative and sum to 1). Moreover, (33) is a system of linear and uncoupled differential equations of the form (11), monotonically converging towards $\sum_{D^{\prime} \in R(D)} \alpha_{D^{\prime}} \phi\left(D^{\prime}\right)=\phi$.

We state without proof that the above monotonicity properties also hold for the approximation $\Psi(D)$ of $\Phi(D)$, given the corresponding redefinition of $\boldsymbol{H}(\boldsymbol{x})$ (Section 6.2). These monotonicity properties are used in the proof of Proposition 6.4.

\section{References}

[1] R. Alur, C. Belta, F. Ivančíc, V. Kumar, M. Mintz, G.J. Pappas, H. Rubin, and J. Schlug. Hybrid modeling and simulation of biomolecular networks. In M.D. Di Benedetto and A. Sangiovanni-Vincentelli, editors, Hybrid Systems: Computation and Control (HSCC 2001), volume 2034 of LNCS, pages 19-32. Springer-Verlag, 2001.

[2] R. Alves and M.A. Savageau. Comparing systemic properties of ensembles of biological networks by graphical and statistical methods. Bioinformatics, 16(6):527-533, 2000.

[3] R. Alves and M.A. Savageau. Systemic properties of ensembles of metabolic networks: Application of graphical and statistical methods to simple unbranched pathways. Bioinformatics, 16(6):534-547, 2000.

[4] N. Barkai and S. Leibler. Robustness in simple biochemical networks. Nature, 387(6636):913-917, 1997.

[5] A. Becskei and L. Serrano. Engineering stability in gene networks by autoregulation. Nature, 405(6786):590-591, 2000. 
[6] A. Ben-Hur and H.T. Siegelmann. Computation in gene networks. In M. Margenstern and Y. Rogozhin, editors, Machines, Computations, and Universality: Third International Conference (MCU 2001), volume 2055 of LNCS, pages 1124. Springer-Verlag, Berlin, 2001.

[7] J.L. Cherry and F.R. Adler. How to make a biological switch. J. Theor. Biol., 203(2):117-133, 2000.

[8] H. de Jong. Modeling and simulation of genetic regulatory systems: A literature review. J. Comput. Biol., 9(1):69-105, 2002.

[9] H. de Jong, J. Geiselmann, G. Batt, C. Hernandez, and M. Page. Qualitative simulation of the initiation of sporulation in B. subtilis. Technical Report RR4527, INRIA, 2002. Submitted to Bull. Math. Biol.

[10] H. de Jong, J. Geiselmann, C. Hernandez, and M. Page. Genetic Network Analyzer: Qualitative simulation of genetic regulatory networks. Bioinformatics, 19(3):336-344, 2003.

[11] H. de Jong, J.-L. Gouzé, C. Hernandez, M. Page, T. Sari, and H. Geiselmann. Qualitative simulation of genetic regulatory networks using piecewise-linear models. Technical Report RR-4407, INRIA, 2002.

[12] H. de Jong, M. Page, C. Hernandez, and J. Geiselmann. Qualitative simulation of genetic regulatory networks: Method and application. In B. Nebel, editor, Proc. Seventeenth Int. Joint Conf. Artif. Intell., IJCAI-01, pages 67-73, San Mateo, CA, 2001. Morgan Kaufmann.

[13] C. Edwards and S.K. Spurgeon. Sliding Mode Control: Theory and Applications. Taylor \& Francis, 1998.

[14] R. Edwards. Analysis of continuous-time switching networks. Physica D, 146:165-199, 2000.

[15] R. Edwards and L. Glass. Combinatorial explosion in model gene networks. Chaos, 10(3):691-704, 2000.

[16] R. Edwards, H.T. Siegelmann, K. Aziza, and L. Glass. Symbolic dynamics and computation in model gene networks. Chaos, 11(1):160-169, 2001.

[17] M.B. Elowitz and S. Leibler. A synthetic oscillatory network of transcriptional regulators. Nature, 403(6767):335-338, 2000.

[18] D. Endy and R. Brent. Modelling cellular behavior. Nature, 409(6818):391-395, 2001.

[19] A.F. Filippov. Differential Equations with Discontinuous Righthand Sides. Kluwer Academic Publishers, 1988. 
[20] K.D. Forbus. Qualitative process theory. Artif. Intell., 24:85-168, 1984.

[21] T.S. Gardner, C.R. Cantor, and J.J. Collins. Construction of a genetic toggle switch in Escherichia coli. Nature, 403(6786):339-342, 2000.

[22] R. Ghosh, A. Tiwari, and C.L. Tomlin. Automated symbolic reachability analysis with application to Delta-Notch signaling automata. In A. Pnueli and O. Maler, editors, Hybrid Systems: Computation and Control (HSCC 2003), volume 2623 of LNCS, pages 233-248. Springer-Verlag, Berlin, 2003.

[23] R. Ghosh and C.J. Tomlin. Lateral inhibition through Delta-Notch signaling: A piecewise affine hybrid model. In M.D. Di Benedetto and A. SangiovanniVincentelli, editors, Hybrid Systems: Computation and Control (HSCC 2001), volume 2034 of LNCS, pages 232-246. Springer-Verlag, Berlin, 2001.

[24] L. Glass. Classification of biological networks by their qualitative dynamics. $J$. Theor. Biol., 54(1):85-107, 1975.

[25] L. Glass. Combinatorial and topological methods in nonlinear chemical kinetics. J. Chem. Phys., 63(4):1325-1335, 1975.

[26] L. Glass. Global analysis of nonlinear chemical kinetics. In B. Berne, editor, Statistical Mechanics, Part B: Time Dependent Processes, pages 311-349. Plenum Press, 1977.

[27] L. Glass and S.A. Kauffman. The logical analysis of continuous non-linear biochemical control networks. J. Theor. Biol., 39(1):103-129, 1973.

[28] L. Glass and J.S. Pasternack. Prediction of limit cycles in mathematical models of biological oscillations. Bull. Math. Biol., 40:27-44, 1978.

[29] L. Glass and J.S. Pasternack. Stable oscillations in mathematical models of biological control systems. J. Math. Biol., 6:207-223, 1978.

[30] B.C. Goodwin. Temporal Organization in Cells. Academic Press, 1963.

[31] J.-L. Gouzé and T. Sari. A class of piecewise linear differential equations arising in biological models. Dynamical Systems, 17(4):299-316, 2003.

[32] K.R. Heidtke and S. Schulze-Kremer. Design and implementation of a qualitative simulation model of $\lambda$ phage infection. Bioinformatics, 14(1):81-91, 1998.

[33] P.D. Karp. Design methods for scientific hypothesis formation and their application to molecular biology. Mach. Learn., 12(12):89-116, 1993.

[34] S.A. Kauffman. The Origins of Order: Self-Organization and Selection in Evolution. Oxford University Press, 1993. 
[35] A.D. Keller. Specifying epigenetic states with autoregulatory transcription factors. J. Theor. Biol., 170(2):175-181, 1994.

[36] A.D. Keller. Model genetic circuits encoding autoregulatory transcription factors. J. Theor. Biol., 172(2):169-185, 1995.

[37] J.L. Kelley. General Topology. Van Nostrand, 1969.

[38] K.W. Kohn. Molecular interaction maps as information organizers and simulation guides. Chaos, 11(1):1-14, 2001.

[39] B. Kuipers. Qualitative Reasoning: Modeling and Simulation with Incomplete Knowledge. MIT Press, 1994.

[40] B.J. Kuipers. Qualitative reasoning: Modeling and simulation with incomplete knowledge. Automatica, 25(4):571-585, 1989.

[41] J.E. Lewis and L. Glass. Steady states, limit cycles, and chaos in models of complex biological networks. Int. J. Bifurcat. Chaos, 1(2):477-483, 1991.

[42] J.E. Lewis and L. Glass. Nonlinear dynamics and symbolic dynamics of neural networks. Neural Comput., 4:621-642, 1992.

[43] D.J. Lockhart and E.A. Winzeler. Genomics, gene expression and DNA arrays. Nature, 405(6788):827-836, 2000.

[44] H.H. McAdams and A. Arkin. Simulation of prokaryotic genetic circuits. Ann. Rev. Biophys. Biomol. Struct., 27:199-224, 1998.

[45] H.H. McAdams and L. Shapiro. Circuit simulation of genetic networks. Science, 269(5224):650-656, 1995.

[46] L. Mendoza, D. Thieffry, and E.R. Alvarez-Buylla. Genetic control of flower morphogenesis in Arabidopsis thaliana: A logical analysis. Bioinformatics, 15(7-8):593-606, 1999.

[47] T. Mestl, E. Plahte, and S.W. Omholt. A mathematical framework for describing and analysing gene regulatory networks. J. Theor. Biol., 176(2):291-300, 1995.

[48] T. Mestl, E. Plahte, and S.W. Omholt. Periodic solutions in systems of piecewise-linear differential equations. Dyn. Stabil. Syst., 10(2):179-193, 1995.

[49] M. Morohashi, A.E. Winn, M.T. Borisuk, H. Bolouri, J. Doyle, and H. Kitano. Robustness as a measure of plausibility in models of biochemical networks. $J$. Theor. Biol., 216(1):19-30, 2002.

[50] S.W. Omholt, X. Kefang, Ø. Andersen, and E. Plahte. Description and analysis of switchlike regulatory networks exemplified by a model of cellular iron homeostasis. J. Theor. Biol., 195(3):339-350, 1998. 
[51] A. Pandey and M. Mann. Proteomics to study genes and genomes. Nature, 405(6788):837-846, 2000.

[52] E. Plahte, T. Mestl, and S.W. Omholt. Global analysis of steady points for systems of differential equations with sigmoid interactions. Dyn. Stabil. Syst., 9(4):275-291, 1994.

[53] E. Plahte, T. Mestl, and S.W. Omholt. Stationary states in food web models with threshold relationships. J. Biol. Syst., 3(2):569-577, 1995.

[54] E. Plahte, T. Mestl, and S.W. Omholt. A methodological basis for description and analysis of systems with complex switch-like interactions. J. Math. Biol., 36(4):321-348, 1998.

[55] E.I. Prokudina, R.Y. Valeev, and R.N. Tchuraev. A new method for the analysis of the dynamics of the molecular genetic control systems. II. Application of the method of generalized threshold models in the investigation of concrete genetic systems. J. Theor. Biol., 151(1):89-110, 1991.

[56] M. Ptashne. A Genetic Switch: Phage $\lambda$ and Higher Organisms. Cell Press \& Blackwell Science, Cambridge, MA, 2nd edition, 1992.

[57] B. Ren, F. Robert, J.J. Wyrick, O. Aparicio, E.G. Jennings I. Simon, J. Zeitlinger, J. Schreiber, N. Hannett, E. Kanin, T.L. Volkert, C.J. Wilson, S.P. Bell, and R.A. Young. Genome-wide location and function of DNA binding proteins. Science, 290(5500):2306-2309, 2000.

[58] L. Sánchez and D. Thieffry. A logical analysis of the Drosophila gap genes. J. Theor. Biol., 211(2):115-141, 2001.

[59] M. Santillán and M.C. Mackey. Dynamic regulation of the tryptophan operon: A modeling study and comparison with experimental data. Proc. Natl. Acad. Sci. USA, 98(4):1364-1369, 2001.

[60] E.H. Snoussi. Qualitative dynamics of piecewise-linear differential equations: A discrete mapping approach. Dyn. Stabil. Syst., 4(3-4):189-207, 1989.

[61] E.H. Snoussi and R. Thomas. Logical identification of all steady states: The concept of feedback loop characteristic states. Bull. Math. Biol., 55(5):973-991, 1993.

[62] R. Somogyi and C.A. Sniegoski. Modeling the complexity of genetic networks: Understanding multigenic and pleiotropic regulation. Complexity, 1(6):45-63, 1996.

[63] R.N. Tchuraev and V.A. Ratner. A continuous approach with threshold characteristics for simulation of gene expression. In K. Bellmann, editor, Molecular Genetic Information Systems: Modelling and Simulation, pages 64-80. Akademie-Verlag, 1983. 
[64] D. Thieffry, A.M. Huerta, E. Pérez-Rueda, and J. Collado-Vides. From specific gene regulation to genomic networks: A global analysis of transcriptional regulation in Escherichia coli. BioEssays, 20(5):433-440, 1998.

[65] D. Thieffry and R. Thomas. Dynamical behaviour of biological networks: II. Immunity control in bacteriophage lambda. Bull. Math. Biol., 57(2):277297, 1995.

[66] R. Thomas and R. d'Ari. Biological Feedback. CRC Press, 1990.

[67] R. Thomas, D. Thieffry, and M. Kaufman. Dynamical behaviour of biological regulatory networks: I. Biological role of feedback loops and practical use of the concept of the loop-characteristic state. Bull. Math. Biol., 57(2):247-276, 1995.

[68] R.B. Trelease, R.A. Henderson, and J.B. Park. A qualitative process system for modeling NF- $\kappa \mathrm{B}$ and AP-1 gene regulation in immune cell biology research. Artif. Intell. Med., 17(3):303-321, 1999.

[69] G. von Dassow, E. Meir, E.M. Munro, and G.M. Odell. The segment polarity network is a robust developmental module. Nature, 406(6792):188-192, 2000.

[70] D.M. Wolf and F.H. Eeckman. On the relationship between genomic regulatory element organization and gene regulatory dynamics. J. Theor. Biol., 195(2):167$186,1998$.

[71] G. Yagil and E. Yagil. On the relation between effector concentration and the rate of induced enzyme synthesis. Biophys. J., 11(1):11-27, 1971.

[72] H. Zhu and M. Snyder. Protein arrays and microarrays. Curr. Opin. Chem. Biol., 5(1):40-45, 2001. 